\title{
Radio pulsars: the search for truth
}

\author{
V S Beskin, Ya N Istomin, A A Philippov \\ P N Lebedev Physical Institute, Leninskii prosp. 53, Moscow, 119991, \\ Moscow Institute of Physics and Technology, Institutsky per, 9, Dolgoprudny, Moscow region, 141700, \\ Department of Astrophysical Sciences, Princeton University, 4 Ivy Lane, Peyton Hall, Princeton, NJ 08544 \\ Usp. Fiz. Nauk 183, 179-194 (2013) [in Russian] \\ English translation: Physics - Uspekhi, 56, 164-179 (2013) \\ Translated by G Pontecorvo; edited by A Semikhatov
}

\begin{abstract}
It was as early as the 1980s that A V Gurevich and his group proposed a theory to explain the magnetosphere of radio pulsars and the mechanism by which they produce coherent radio emission. The theory has been sharply criticized and is currently rarely mentioned when discussing the observational properties of radio pulsars, even though all the criticisms were in their time disproven in a most thorough and detailed manner. Recent results show even more conclusively that the theory has no internal inconsistencies. New observational data also demonstrate the validity of the basic conclusions of the theory. Based on the latest results on the effects of wave propagation in the magnetosphere of a neuron star, we show that the developed theory does indeed allow quantitative predictions of the evolution of neutron stars and the properties of the observed radio emission.
\end{abstract}

\section{Contents}

\section{Introduction}

\section{Pulsar magnetosphere}

2.1 Screening of the magnetodipole radiation

2.2 Current losses . . . . . . . . . . . . .

2.3 Evolution of the inclination angle . . . .

2.4 Light surface . . . . . . . . . . .

3 Theory of radio emission

3.1 Formulation of the problem . . . . . . . 9

3.2 Polarization of the curvature wave . . . . 9

3.3 Propagation of the triplet of harmonics . . 10

3.4 Calculation of the dielectric permittivity tensor ............. 13

3.5 Propagation theory of radio waves . . . 15

4 Conclusion

\section{Introduction}

Thirty years have passed since the group led by A V Gurevich at the Theoretical Physics Department of the Lebedev Physical Institute, Russian Academy of Sciences, published its first article [1] on the theory of the magnetosphere of radio pulsars, 25 years since the publication of their article [2] dealing with the development of the theory of radio emission of pulsars, and, finally, 20 years since the publication of mongraph [3], in which a consistent theory was formulated of the principal processes responsible for the observed activity of radio pulsars. In hindsight, we would like to make some comments, which, we hope, can be useful at the present stage of development of the theory.

Currently, already more than 40 years since radio pulsars were discovered [4] in 1967, our understanding of these objects remains somewhat ambiguous. On the one hand, significant progress had been achieved already during the first decade after the discovery of radio pulsars, when the theory of the magnetosphere of radio pulsars and the theory of their radio emission were being actively developed by leading scientists throughout the world: 1 Ginzburg [5], Zheleznyakov [6], Kadomtsev [7], Sagdeev [8], and Lominadze [9] in the USSR, and Goldreich [10],
Total success was not achieved, however, in spite of a as also was the importance of the copreciated $[14,17]$, as also was the importance of the current mechanism of energy losses, i.e., of energy losses due to longitudinal currents flowing in the magnetosphere of a pulsar [10]). In particular, it is only in recent years that processes occurring in the magnetosphere of an oblique rotator have 
started to be actively discussed [18-21]; previously, most work was devoted to the axisymmetric magnetosphere [22-29]. No common standpoint regarding the mechanism of coherent radio emission exists yet [30-32].

By the late 1980s, research activities on the theory of the pulsar magnetosphere and the theory of radio emission were drastically reduced. Two or three serious publications per year (!) certainly made no real difference. Actually, a period of deep stagnation set in. On the one hand, most theorists were not able to propose a model that could provide readily testable predictions, while, on the other hand, the existence of a simple hollow-cone geometric model [33] (in which the directivity pattern of the radio emission repeats the density profile of the secondary electron-positron plasma outflowing along the open magnetic field lines) permitted interpreting observational data without turning to theory. As a result, the connection between theory and observations was practically lost.

Perhaps, precisely the atmosphere of general failure (although there most likely existed purely opportunistic reasons, also) resulted in a series of studies, performed in the 1980 s by the group led by Gurevich $[1,2,34,35]$, in which the authors succeeded in formulating a consistent theory of the magnetosphere and of the radio emission of pulsars, which was met, mildly speaking, without friendliness. Here, we only present a few quotations from articles and reviews on book [3] that was the conclusion of a decade of work. "We conclude that their computation of the dielectric tensor of a plasma in a strong magnetic field is wrong" [38]. "It has been claimed that this instability is spurious" [39]. "This theory is known to be incorrect. It contains several fatal flows" [40].

Regretfully, such peremptory criticism made any serious discussion of the results of our work simply impossible, although practically all the main critical pronouncements were given detailed explanations $[30,41$, 42], demonstrating their judgements to be unjustified. Therefore, as before, we believe in the validity of our conclusions, which can be formulated as follows.

\section{Theory of the magnetosphere of radio pulsars.}

1. The plasma filling the pulsar magnetosphere totally screens the magnetodipole radiation of a neutron star. As a result, all the energy losses must be due to longitudinal currents circulating within the pulsar magnetosphere (and closing up on the surface of the neutron star).

2. For a local Goldreich current (see Section 2.2), current losses should be significantly smaller for an orthogonal rotator than for the axisymmetric one, which follows from the expression we found for current losses for an arbitrary inclination angle of the magnetic dipole axis to the axis of rotation.

\footnotetext{
${ }^{1}$ Incidentally, the fact that critical reviews continued to appear until quite recently $[36,37]$ rather serves as an argument in favor of our conclusions.
}

3. The inclination angle of the magnetic dipole axis to the axis of rotation should increase with time, unlike the magnetodipole losses.

4. Quite a small longitudinal current is realized in the pulsar magnetosphere, which results in the inevitable appearance outside the light cylinder of a region where the electric field is greater than the magnetic field. Inside this region, effective acceleration of the plasma flowing out of the pulsar magnetosphere becomes possible.

II. Theory of radio emission.

1. The dielectric permittivity tensor of plasma in an inhomogeneous medium has been found, in particular, for the relativistic plasma moving along curved magnetic field lines. This tensor permits correctly describing the interaction of particles with waves propagating in an inhomogeneous plasma.

2. The analysis of such a tensor reveals the instability of 'curvature-plasma' waves, which can serve as the base instability for the maser mechanism providing coherence of the observed emission.

3. By taking the nonlinear interaction of waves into account, the excitation level has been determined for transverse waves capable to escape from the magnetosphere of a neutron star, and, thus, the intensity of the radio emission of pulsars has been established.

4. Based on a consistent analysis of wave propagation in the magnetosphere of a pulsar, which, for instance, takes the refraction of an ordinary wave into account, quantitative predictions have been made concerning the main observational characteristics of pulsars (the frequency dependence of the width of mean profiles, the statistics of pulsars with single or double profiles, etc.).

The purpose of this article is to show that at present, sufficient material has been accumulated to assert with confidence that the principal theoretical points of our theory not only have not become obsolete (which could well have happened owing to the impetuous development of numerical methods) but also can be a basis for understanding the phenomenon of radio pulsars. Moreover, we show that the recently obtained observational data confirm the validity of the main theoretical conclusions formulated over 20 years ago.

\section{Pulsar magnetosphere}

\subsection{Screening of the magnetodipole ra- diation}

A uniformly magnetized sphere rotating in the vacuum is known to lose rotational energy owing to magnetodipole losses [43]:

$$
W_{\text {tot }}=-J_{\mathrm{r}} \Omega \dot{\Omega}=\frac{1}{6} \frac{B_{0}^{2} \Omega^{4} R^{6}}{c^{3}} \sin ^{2} \chi .
$$


Here, $R$ is the radius of the sphere, $B_{0}$ is the magnetic field at the magnetic pole, $J_{\mathrm{r}} \sim M R^{2}$ is the moment of inertia, $\chi$ is the inclination angle of the magnetic axis to the rotation axis, and $\Omega=2 \pi / P$ is the angular velocity of rotation. This mechanism is quite universal, and hence it would be natural to assume expression (1) for magnetodipole losses to also hold in the case of a magnetosphere filled with secondary electron-positron plasma. Therefore, estimate (1) still expresses the common view of the rotation deceleration mechanism of radio pulsars.

However, this conclusion, seemingly evident at first glance, turned out to have no foundation. To be more precise, we showed that in the framework of a forcefree approximation (a secondary plasma, whose energy density is negligible compared to the energy density of the magnetic field, fully screens the longitudinal electric field) and in the case of a zero longitudinal (parallel to the magnetic field) electric current, the flux of the Poynting vector through the surface of a light cylinder, $R_{\mathrm{L}}=c / \Omega$, vanishes [1]. From a mathematical standpoint, this is because the toroidal component of the magnetic field on the surface of the light cylinder must vanish (actually, this conclusion was obtained in 1975 in Ref. $[44])$ :

$$
B_{\varphi}\left(R_{\mathrm{L}}\right)=0 .
$$

From a physical standpoint, this means that the plasma filling the pulsar magnetosphere completely screens the magnetodipole radiation of the neutron star. In other words, in the case of a zero longitudinal current, the magnetospheric plasma emission is in a phase precisely opposite to the phase of the pulsar magnetodipole radiation. Consequently, all the energy losses should be due to longitudinal currents circulating inside the magnetosphere of the neutron star and closing up on its surface. These energy losses can be determined by the formula $W_{\text {tot }}=-\boldsymbol{\Omega K}$, where

$$
\mathbf{K}=\frac{1}{c} \int\left[\mathbf{r} \times\left[\mathbf{J}_{\mathrm{s}} \times \mathbf{B}\right]\right] \mathrm{d} S
$$

is the decelerating moment of the Ampere force due to surface currents $J_{\mathrm{s}}$. We recall that it is possible to obtain an analytic solution for an oblique rotator because in the case of a zero longitudinal current, the equation describing the magnetosphere of a neutron star is linear; it is also extremely important here that the boundary condition at infinity (along the rotation axis) was used.

It follows that the energy losses can be written as [1]

$$
W_{\mathrm{tot}}=\frac{f_{*}^{2}}{4} \frac{B_{0}^{2} \Omega^{4} R^{6}}{c^{3}} i_{0} \cos \chi,
$$

where $i_{0}=j_{\|} / j_{\mathrm{GJ}}$ is the dimensionless longitudinal current normalized to the so-called Goldreich current (or the Golreich-Julian current),

$$
j_{\mathrm{GJ}}=\frac{\Omega B}{2 \pi},
$$

where $f_{*}=1.59-1.96$ is a coefficient that depends weakly on the inclination angle $\chi$.
We note that the conclusion concerning the complete screening of the magnetodipole radiation was, naturally, not obvious. Therefore, not surprisingly, it remains far from being adopted by everyone. It is interesting that now, after 30 years have passed, we have been surprised to learn from many participants in those discussions that the main criticism seems to have concerned our alleged claim that pulsars lose no rotation energy at all. But we never made any such statement and could not have done so. The main conclusion in Ref. [1] is formula (4) for current energy losses, which clearly points to the deceleration mechanism.

At present, screening of the magnetodipole radiation of a neutron star can be confidently said to indeed take place. First of all, in 1999, the group of L Mestel [45] performed studies equivalent to those presented in Ref. [1] and fully confirmed our conclusions: in the case of a zero longitudinal current, the energy losses of an oblique rotator are equal to zero. Fig. 1 shows the structure of the magnetic field of an orthogonal rotator in the equatorial plane, obtained in Ref. [45] (the corresponding cross section remained in the draft copies of Ref. [1]). It is clearly seen that the magnetic field lines indeed approach the light cylinder at a right angle.

However, the most important recent result is that magnetodipole losses are also absent in the solution for the magnetosphere of an oblique rotator constructed by Spitkovsky on the basis of numerical simulation [20]. First of all, this follows from the assertion concerning the split-monopole asymptotic form of the solution obtained, which is close to the model of radial magnetized wind [18]. Such flows only involve stationary magnetized wind (independent of time outside a thin current sheet), in which, however, the energy flux is related to the flux of the Poynting vector. But the main point is that the absence of magnetodipole losses is also confirmed by a straightforward analysis of the structure of electromagnetic fields in Ref. [20].

Indeed, in the case of a vacuum rotator, for any inclination angle $\chi \neq 0^{\circ}$, a variable-in-time component of the magnetic field must be present on the rotation axis, with the amplitude

$$
B_{\perp}=\frac{|\ddot{\mathbf{m}}|}{c^{2} r},
$$

where $\mathbf{m}$ is the magnetic moment of the star (with $\left.|\mathbf{m}|=B_{0} R^{3} / 2\right), \ddot{\mathbf{m}}$ is its second time derivative, and $|\ddot{\mathbf{m}}|=|\mathbf{m}| \Omega^{2} \sin \chi$. However, as can be seen from Fig. 2 , the variable component of the magnetic field in the Spitkovsky solution decreases much more rapidly, like $1 / r^{3}$. In our opinion, the absence of variable fields decreasing as $1 / r$ in the numerical solution for an oblique rotator is the most convincing proof of the total screening of magnetodipole radiation in the case of a magnetosphere completely filled with plasma.

\subsection{Current losses}

One more important consequence of the theory of current losses is that for a local longitudinal Goldreich cur- 


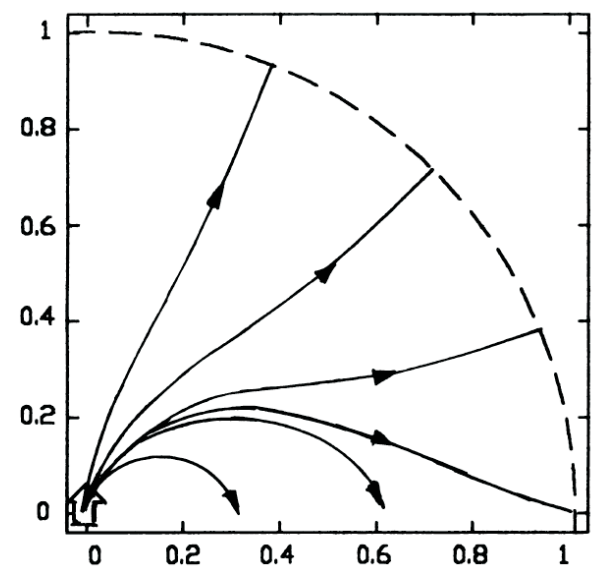

Figure 1: Structure of magnetic field lines of an orthogonal rotator in the equatorial plane [45]. The toroidal magnetic field is zero on the light cylinder.

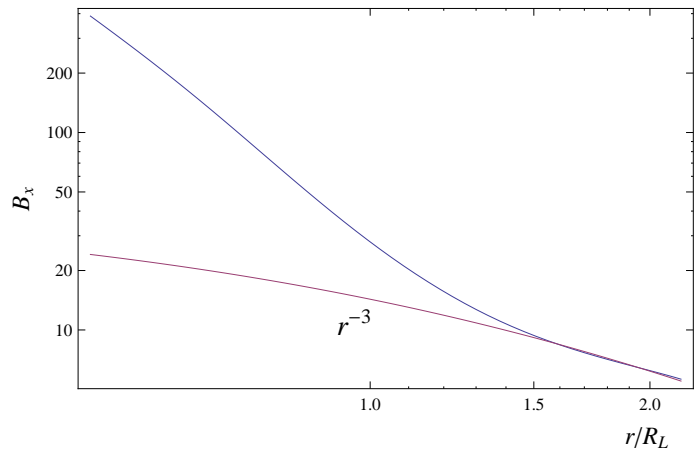

Figure 2: Dependence of the $B_{x}$ component of the magnetic field on the rotation axis on the distance $r$ from the neutron star in numerical simulation [20]. The lower curve shows the asymptotic behaveour $B \propto r^{-3}$. The inclination angle is $\chi=60^{\circ}$.

rent, the rotational energy losses should decrease as the inclination angle $\chi$ increases $[1,3]$. The point is that, besides the factor $\cos \chi$ related to the scalar product $W_{\text {tot }}=-\boldsymbol{\Omega} \cdot \mathbf{K}$, a significant dependence of the current losses $W_{\text {tot }}$ in Eqn (4) on the inclination angle is also involved in the quantity $i_{0}$.

Indeed, in the definition of the dimensionless current $i_{0}=j_{\|} / j_{\mathrm{GJ}}$, the denominator contains the Goldreich current for the axisymmetric case, while in the case of nonzero angles $\chi$, the Goldreich-Julian charge density in the vicinity of the magnetic poles

$$
\rho_{\mathrm{GJ}}=-\frac{\Omega \mathbf{B}}{2 \pi c} \approx-\frac{\Omega B}{2 \pi c} \cos \chi
$$

itself depends on the angle $\chi$. On the other hand, it is natural to expect the longitudinal current to be bounded by the value $j_{\|} \approx \rho_{\mathrm{GJ}} c$. At any rate, both in the
Ruderman-Sutherland model [14] with the particle escape from the surface of a neutron star being hindered and in the Arons model [46], in which the escape of particles is free, the longitudinal current is indeed determined by the relation $j_{\|} \approx \rho_{\mathrm{GJ}} c$.

But then, in the case of an oblique rotator, the dimensionless current $i_{0}$ has the upper bound $i_{0}^{(\max )}(\chi) \sim$ $\cos \chi$. As a result, the current losses must decrease as the angle $\chi$ increases, at least like $\cos ^{2} \chi$. In particular, if $\chi=90^{\circ}$ (when $\cos ^{2} \chi$ is to be substituted by its characteristic value in the range of the polar cap, $\left.<\cos ^{2} \chi>\approx \Omega R / c\right)$, we obtain

$$
W_{\mathrm{tot}}=c_{\perp} \frac{B_{0}^{2} \Omega^{4} R^{6}}{c^{3}}\left(\frac{\Omega R}{c}\right) i_{\mathrm{A}} .
$$

In the case of a local Goldreich current, $i_{\mathrm{A}}=1$, while the coefficient $c_{\perp} \sim 1$ already depends not only on the profile of the asymmetric longitudinal current but also on the shape of the polar cap.

In discussions of this issue, the following reasoning is standardly used as an argument against the decrease in losses occurring as $\chi$ increases. In expression (3) for the decelerating moment, an increase in the angle $\chi$ is indeed accompanied by the surface current $J_{\mathrm{s}}$ decreasing as $\cos \chi$. But the characteristic distance between the axis and the polar cap increases as $\sin \chi$, and hence, even in the case of a local Goldreich current, the losses depend weakly on the inclination angle.

However, as has been demonstrated by a more precise analysis in Ref. [1], the above reasoning, which seems obvious at first glance, does not take the real structure of surface currents in the polar cap region into account. As shown in Fig. 3, the currents that close up should actually be arranged such that the current averaged over the polar cap surface vanish. Consequently, in determining the deceleration rate of a radio pulsar, it is necessary to consider higher-order effects, such as the effect of the curved surface of a neutron star.

But if the averaged surface current within the polar cap indeed vanishes, then, as shown in Fig. 3, a surface current comparable in value to the volume current flowing in the magnetosphere should flow along the separatrix separating the regions of closed and open field lines. In the case of an orthogonal rotator (and for a circular polar cap, when the result can be obtained analytically), the inverse current should amount to $3 / 4$ of the volume current flowing in the region of open field lines. A remarkable event was that numerical simulation [47] of the magnetosphere of an oblique rotator actually revealed inverse currents flowing along the separatrix. True, the inverse current only amounted to $20 \%$ of the volume current. But such a discrepancy between the results of simulation and theoretical predictions can most likely be explained by the radius of the star being set in simulations only to half the radius of the light cylinder, when the magnetic field near the magnetic poles already differs strongly from the dipole field.

Finally, we note that no contradiction actually exists, 


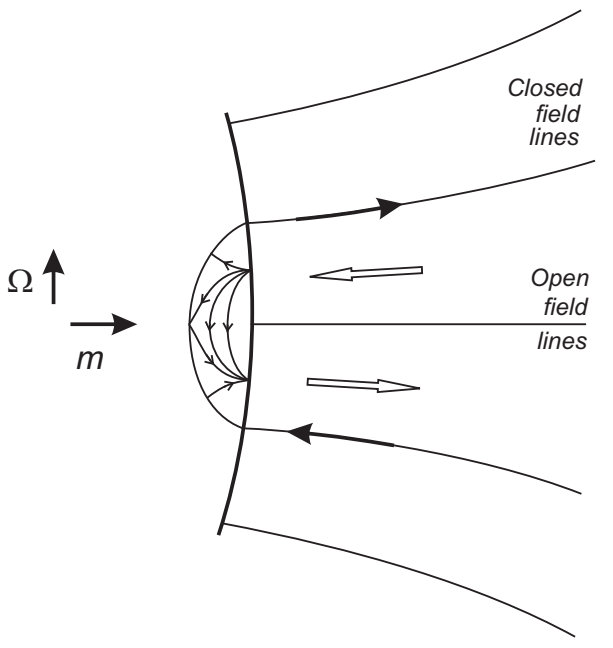

Figure 3: Structure of electric currents flowing in the vicinity of the magnetic poles of an orthogonal rotator. The currents flowing along separatices (bold arrows), separating the regions of closed and open field lines, close the longitudinal volume currents (contoured arrows) such that surface currents are fully concentrated within the polar cap.

either, between relation (4) and the expression

$$
W_{\mathrm{tot}} \approx \frac{1}{4} \frac{B_{0}^{2} \Omega^{4} R^{6}}{c^{3}}\left(1+\sin ^{2} \chi\right)
$$

obtained by Spitkovsky for an oblique rotator; approximate formula (9) was obtained in Ref. [20] for a magnetosphere in which the longitudinal current was actually significantly larger than the local Goldreich current (see Ref. [48] for the details), which is consistent with the condition $i_{0}>1$ (correspondingly, $i_{\mathrm{A}}>1$ ). A longitudinal current exceeding the local Goldreich current was necessary for constructing a smooth solution containing the magnetohydrodynamic (MHD) wind outflowing to infinity (see Section 2.4).

It is interesting that one more possibility was recently revealed for directly testing the validity of expression (4) for current losses $W_{\text {tot }}$ (and at the same time the validity of the assertion that the pulsar magnetosphere completely screens the magnetodipole radiation of a neutron star). The possibility of implementing such a test is related to the unusual properties of the pulsar B1931+24 [49]. Unlike the radiation of other radio pulsars, the radiation of B1931+24 is strongly variable. This pulsar is in an active state for 5-10 days, then its radio emission is switched off in less than $10 \mathrm{~s}$, and it is no longer observable for the next 25-30 days. It is important that the absolute value of the rotation deceleration of B1931+24 is different in the 'on' and 'off' states:

$$
\begin{aligned}
& \dot{\Omega}_{\text {on }}=-1.02 \times 10^{-14}\left[\mathrm{~s}^{-2}\right], \\
& \dot{\Omega}_{\text {off }}=-0.68 \times 10^{-14}\left[\mathrm{~s}^{-2}\right],
\end{aligned}
$$

with

$$
\frac{\dot{\Omega}_{\mathrm{on}}}{\dot{\Omega}_{\mathrm{off}}} \approx 1.5
$$

Later, the pulsar J1832+0031 was also found to exhibit similar behavior $\left(t_{\mathrm{on}} \sim 300\right.$ days, $t_{\mathrm{off}} \sim 700$ days, and in this case, also, the ratio $\dot{\Omega}_{\text {on }} / \dot{\Omega}_{\text {off }} \approx 1.5$ ), as did the pulsar J1841-0500 (in that case, $\dot{\Omega}_{\text {on }} / \dot{\Omega}_{\text {off }} \approx 2.5[50]$ ).

It is natural to assume that the difference between $\dot{\Omega}_{\text {on }}$ and $\dot{\Omega}_{\text {off }}$ for these pulsars occurs simply because deceleration in the switched-on state is related to current losses, while in the switched-off state (when the magnetosphere is not filled with plasma), it is due to the emission of a magnetodipole wave $[51,52]$. Then, using relations (1) and (4), we obtain

$$
\frac{\dot{\Omega}_{\mathrm{on}}}{\dot{\Omega}_{\mathrm{off}}}=\frac{3 f_{*}^{2}}{2} \cot ^{2} \chi
$$

which yields a reasonable value for the inclination angle $\chi \approx 60-70^{\circ}$. On the other hand, if relation (9) is applied for the switched-on state, we arrive at

$$
\frac{\dot{\Omega}_{\mathrm{on}}}{\dot{\Omega}_{\mathrm{off}}}=\frac{3}{2} \frac{\left(1+\sin ^{2} \chi\right)}{\sin ^{2} \chi} .
$$

Clearly, this quantity cannot be equal to 1.5 or 2.5 for any value of the inclination angle 2 . If such an interpretation of the observations corresponds to reality, it follows that the longitudinal current flowing in the magnetosphere does not actually exceed the local Goldreich current.

\subsection{Evolution of the inclination angle}

Determining the evolution of the inclination angle $\chi$ could serve as one more test. For current losses (more exactly, for local Goldreich current, and for inclination angle $\chi \neq 90^{\circ}$ ), the decelerating moment $\mathbf{K}$ is directed opposite to the magnetic moment of the neutron star $\mathbf{m}$, and therefore the Euler equation leads to the conservation of the projection of the rotation angular velocity $\boldsymbol{\Omega}$ onto the axis perpendicular to $\mathbf{K}$. Hence, the following quantity must be conserved during the evolution [3]:

$$
\Omega \sin \chi=\text { const. }
$$

Consequently, in the case of current losses, the angle $\chi$ between the axis of rotation and the magnetic axis should increase (but not decrease, as in the case of magnetodipole radiation), and the characteristic time of its evolution should coincide with the characteristic dynamical age of the pulsar, $\tau_{\mathrm{D}}=P / 2 \dot{P}[34]$. Regretfully, no method has been found to determine the direction of evolution of the inclination angle $\chi$ for individual pulsars (see, however, Ref. [54]). On the other hand, the

\footnotetext{
[53].

${ }^{2}$ For this reason, relation (9) was somewhat corrected in Ref.
} 


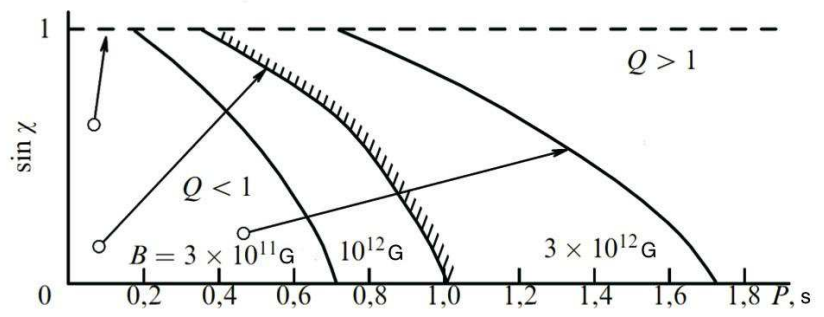

Figure 4: Pulsar extinction line in a $P-\sin \chi$ diagram for different inclination angles $\chi$ and different magnetic fields. Arrows show the evolution tracks of individual pulsars in the model of the current losses (15). The production of secondary particles is suppressed at angles $\chi$ close to $90^{\circ}$. Therefore, neutron stars, which in the diagram are above and to the right of the extinction line, do not manifest themselves as radio pulsars, independently of the deceleration mechanism.

prediction indicating an increase in the angle $\chi$ is known not to contradict observations statistically $[34,55]$.

The last assertion requires clarification. Observations reveal average statistical inclination angles $\chi$ indisputably decrease as the period $P$ of pulsars increases and its derivative $\dot{P}$ decreases [56]. Therefore, the average statistical inclination angle decreases as the dynamic age $\tau_{\mathrm{D}}$ increases. Correspondingly, pulsars with larger periods can be observed to exhibit relatively larger widths of the mean pulses $W_{\mathrm{r}}=W_{\mathrm{r}}^{(0)} / \sin \chi[57]$ (where $W_{\mathrm{r}}^{(0)}$ is the width of thedirectivity pattern). But this by no means implies that the inclination angle for each concrete pulsar decreases with time. Such a behavior of the average inclination angle $\chi$ can also be realized when the angles $\chi$ of each pulsar increase in accordance with (15).

Indeed, as can be seen from Fig. 4, for the given values of the pulsar period $P$ and the magnetic field $B_{0}$, the production of particles is suppressed precisely at angles $\chi$ close to $90^{\circ}$. This is because Goldreich-Julian charge density (7) decreases significantly at such angles, which in turn leads to a decrease in the electric potential drop near the surface of the neutron star. As a result, stable production of secondary particles becomes impossible there. Therefore, owing to such a dependence of the pulsar extinction line on $\chi$, the average inclination angle can also decrease as the dynamic age increases, for example, in the case of pulsars uniformly distributed over the $(P, \sin \chi)$ plane. A detailed analysis, already carried out in Ref. [34] on the basis of a kinetic equation describing the distribution of pulsars (see also later studies $[58,59]$ ) confirmed that the observed distribution of pulsars over the inclination angle does not contradict hypothesis (15) of the increase in the angle $\chi$ for any individual pulsar.

In any case, it is quite clear, that the inclination angle $\chi$ is a key hidden parameter: without taking it into account, it is impossible to construct a consistent theory of the evolution of radio pulsars. Regretfully, with a few ex- ceptions (see, e.g., Ref. [60]), modern theorists (so-called scenario producers) describing the evolution of neutron stars [61-63] do not take the influence of the inclination angle evolution on the observed pulsar distribution into account.

\subsection{Light surface}

Starting from the 1970s, the magnetosphere of a pulsar has been discussed almost exclusively in the forcefree approximation $[23,64-66]$. The reason is that the plasma filling the magnetosphere of a neutron star is less important (secondary) than the magnetic field; therefore (at least within the light cylinder), the particle energy density can be neglected.

In the force-free approximation, the structure of the magnetosphere is described by the so-called pulsar equation, i.e., an elliptic equation for the magnetic flux function. In Section 2.1, in discussing the solution of a similar equation for the zero longitudinal current, we noted that in the case of numerical simulation of an axisymmetric magnetosphere, it is necessary to introduce an additional condition for the external boundary of the integration region [24-29]. Such a condition is usually chosen in the form that the magnetic field lines be radial. In this approach, precisely such an additional condition fixes the longitudinal current flowing within the magnetosphere. Therefore, it is not surprising that the current is close to the critical current $j_{\mathrm{GJ}}=\rho_{\mathrm{GJ}} c(5)$ obtained analytically by F C Michel [64] for the quasispherical wind.

A very important property of this solution is that the energy in the wind is carried by the crossed fields $E_{\theta}$ and $B_{\varphi}$, which form a radial flux of the electromagnetic energy (the Poynting vector flux), and the electric field is smaller than the magnetic field as far as infinity. Otherwise, the freezing-in condition $\mathbf{E}+\mathbf{v} \times \mathbf{B} / c=0$, which always serves as the cornerstone in the approach considered, would be violated.

On the other hand, as is readily verified, implementation of the condition $E<B$ is possible only if the longitudinal electric current $I$ flowing in the magnetosphere is suffiently large. Indeed, in the case of a quasispherical wind outside the light cylinder, the electric field

$$
E_{\theta}=\frac{\Omega r \sin \theta}{c} B_{\mathrm{p}}
$$

and the toroidal magnetic field

$$
B_{\varphi}=\frac{2 I}{c r \sin \theta}
$$

decrease with the distance $r$ as $r^{-1}$ (while the poloidal field $B_{\mathrm{p}}$ decreases as $r^{-2}$ ). Therefore, for the light surface to recede to infinity, it is necessary that the toroidal magnetic field on the light cylinder be of the same order of magnitude as the poloidal field. Implementation of this condition is possible precisely when the total current $I$ outflowing beyond the light cylinder is not less than the Goldreich current $I_{\mathrm{GJ}}=\pi j_{\mathrm{GJ}} R_{0}^{2}$, where $R_{0}$ is the radius of the polar cap. We stress that in all numerical 
simulations, no restrictions were imposed on the longitudinal current outflowing from the neutron star surface. Therefore, it is not surprising that the longitudinal current obtained as a solution of the problem turned out to be of the order of $I_{\mathrm{GJ}}$.

We recall that in the complete MHD version, where taking the finiteness of particle masses into account results in the appearance of an additional critical (fast magnetosonic) surface, the longitudinal current is no longer a free parameter [67]. The value of the longitudinal current is close to $I_{\mathrm{GJ}}$. Therefore, most reseachers currently consider the existence of a strongly magnetized wind for which the condition $|\mathbf{E}|<|\mathbf{B}|$ is satisfied to be practically proven $[20,21]$. We stress that the issue here concerns scales comparable to the radius of the light cylinder $\left(r \sim 1-100 R_{\mathrm{L}}\right)$; at larger scales, as follows, for instance, from an analysis of the interaction of the pulsar wind with supernova remnants [68], the main energy must already be carried by particles. As is known, within the theory of strongly magnetized wind, such an acceleration required for a quasispherical outflow cannot be obtained [67, 69-71].

Generally speaking, the rigorous analytic conclusion that the longitudinal current is close to the critical one only concerned stationary axisymmetric flows. But numerical calculations recently performed for nonstationary force-free configurations [53] have shown unambiguously that the system undergoes quite rapid evolution precisely toward a state with a current close to the critical current. And such a state corresponds to the minimum-energy configuration (for example, minimum energy is exhibited by configurations in which the singular point separating the regions of closed and open field lines is on the light cylinder, but not inside the magnetosphere $[29,72] 3)$. Thus, the existence of quite a strong longitudinal current has been confirmed once again; in any case, no restrictions were imposed on the longitudinal current either.

The following problem arises here, however. As noted, all the theories of stationary particle production in the magnetosphere of a neutron star $[3,14,46,73,74]$ unambiguously testify in favor of the longitudinal current density not possibly being larger than the local Goldreich current, which, as can be seen from its definition (7), depends on the inclination angle $\chi$

$$
j_{\mathrm{GJ}}=\frac{\Omega B}{2 \pi} \cos \chi .
$$

For example, in the case of an orthogonal rotator, the local Goldreich-Julian charge density in the vicinity of the magnetic poles must be $(\Omega R / c)^{1 / 2}$ times smaller than in the case of an axisymmetric magnetosphere. Hence, the longitudinal current flowing along open field lines should also be smaller in the same proportion (for ordinary pulsars with a period $P \sim 1 \mathrm{~s}$, this current is nearly 100 times smaller for an orthogonal rotator). Therefore, in

\footnotetext{
${ }^{3}$ Solutions in which the singular point is inside the light cylinder are, most likely, affected by the limited time available for numerical calculations (A Tchekhovskoy, private communication).
}

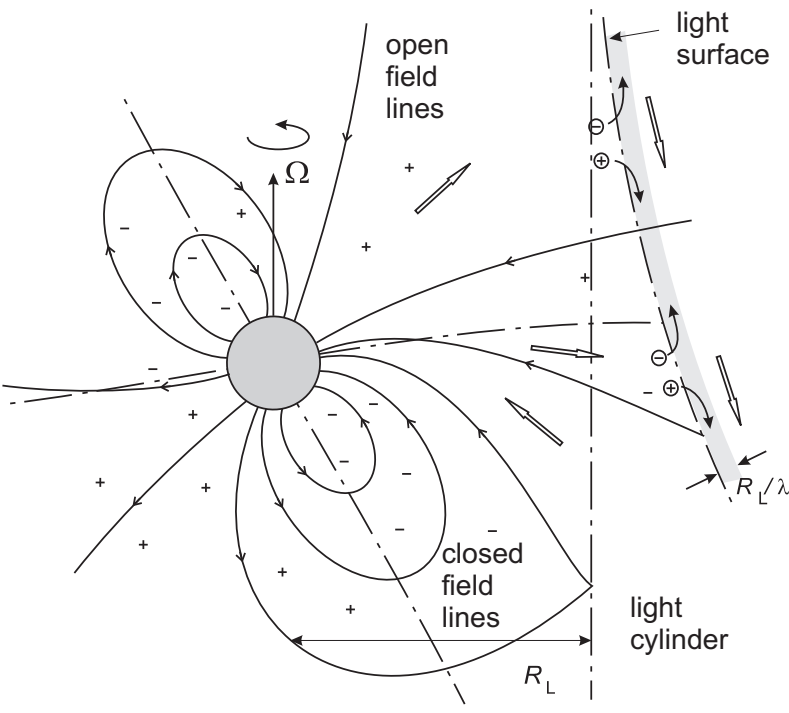

Figure 5: Structure of a magnetosphere with a small enough longitudinal current, having a natural boundary — the 'light surface' $|\mathbf{E}|=|\mathbf{B}|$ at which the freezing-in condition cannot be satisfied. Therefore, electrons and positrons are accelerated in opposite directions along the electric field. Precisely this current closes the longitudinal currents flowing in the magnetosphere.

constructing the solution for an oblique dipole [20], it was necessary to assume the longitudinal current in the region of the magnetic poles to be significantly higher than the local Goldreich current (A Spitkovsky, private communication).

Therefore, the value of the longitudinal current flowing in a neutron star's magnetosphere turns out to be the key issue, without resolving which it is impossible to move toward the understanding of the structure of the magnetosphere of radio pulsars. The problem lies in whether the region of plasma generation at the surface of a neutron star can provide the longitudinal current sufficiently large for the existence of an MHD wind from an oblique rotator. If the necessary current can be created (see, e.g., Ref. [75], where one-dimensional, nonstationary regime was considered), nothing can prevent the production of the MHD wind in which the main part of the energy is carried by the electromagnetic field: such is the opinion of most reseachers. But if the generation of a sufficiently high longitudinal current turns out to be impossible for some reason, then, in the vicinity of the light cylinder, a 'light surface' inevitably arises in which the electric field becomes equal to the magnetic field. Precisely such a structure was predicted by us in Ref. [1].

The appearance of a light surface in the magnetosphere of a radio pulsar radically alters the properties of the pulsar wind because, close to the light surface, closure of the current and effective particle acceleration inevitably occur. Solving the equations of two-fluid hydrodynamics (precisely describing the difference in mo- 
tion between electrons and positrons) in the case of the simplest cylindrical geometry reveals [1] that a significant part of the energy carried within the light surface by the electromagnetic field in the thin transition layer close to the light surface,

$$
\Delta r \sim \lambda^{-1} R_{\mathrm{L}}
$$

is transferred to plasma particles $\left(\lambda=n_{\mathrm{e}} / n_{\mathrm{GJ}} \sim 10^{3}-10^{5}\right.$ is the production multiplicity of particles close to the surface of a neutron star). In the same layer, as shown in Fig. 5, practically total closure of the longitudinal current circulating in the magnetosphere occurs. As a result, a natural explanation is also found for the high efficiency of particle acceleration. Subsequently, a similar result was also obtained for a more realistic geometry, when the poloidal magnetic field near the surface of the light cylinder is close to a monopole field [76]. In particular, it has been confirmed that the particle energy immediately beyond the light surface is by the order of magnitude given by

$$
\begin{aligned}
\mathcal{E}_{\mathrm{e}} & \sim e B_{0} R \frac{1}{\lambda}\left(\frac{\Omega R}{c}\right)^{2} \\
& \sim 10^{4} \mathrm{MeV}\left(\frac{\lambda}{10^{3}}\right)^{-1}\left(\frac{B_{0}}{10^{12} \mathrm{G}}\right)\left(\frac{P}{1 \mathrm{~s}}\right)^{-2},
\end{aligned}
$$

but does not exceed the value $10^{6}$, at which the effects of radiation friction become essential.

Quantity (20) practically corresponds to the total energy transfer from the Poynting vector flux to the flux of accelerated particles. In $(20), \mathcal{E}_{\mathrm{e}}$ is $\lambda$ times smaller than the energy $\mathcal{E}_{\max }=e \Delta V$ corresponding to the maximum potentials difference $\Delta V \sim(\Omega L / c) B L$ of different magnetic field lines in the magnetized wind. Here, $L$ is the characteristic size of the central engine; in radio pulsars, $L$ is equal to the size of the polar cap $R_{0}=(\Omega R / c)^{1 / 2} R$. As a result, we can express the Lorentz factor $\gamma_{\max }=\mathcal{E}_{\mathrm{e}} / m_{\mathrm{e}} c^{2}$ as

$$
\gamma_{\max }=\sigma
$$

where $\sigma$ is the so-called magnetization parameter 4 introduced by Michel [77] in 1969. As was shown in Ref. [48], the magnetization parameter can be represented in the very simple form

$$
\sigma \sim \frac{1}{\lambda}\left(\frac{W_{\mathrm{tot}}}{W_{\mathrm{A}}}\right)^{1 / 2}
$$

where $W_{\text {tot }}$ is the total energy losses and $W_{\mathrm{A}}=m_{\mathrm{e}}^{2} c^{5} / e^{2} \approx 10^{17} \mathrm{erg} \mathrm{s}^{-1}$ is a universal constant; it corresponds to the minimum energy losses of the central engine which can accelerate particles to relativistic energy. Because the particle production multiplicity $\lambda \sim 10^{3}-10^{5}$ for radio pulsars is known

\footnotetext{
${ }^{4}$ Recently, the notation $\mu$ for the magnetization parameter has become popular, with $\sigma$ standing for the ratio of the electromagnetic energy flux to the energy flux carried by particles.
}

$[73,74,78]$, the value of $\sigma$ can also be found.For most pulsars, the value of $\sigma$ lies in the range $10^{3}-10^{4}$, and it can reach $10^{6}$ only in the youngest sources (the Crab and Vela pulsars). We note that the parameter $\sigma$ is very convenient for determining the key parameters of strongly magnetized winds. For example, the radius of the fast magnetsonic surface is expressed simply as

$$
r_{\mathrm{f}} \sim \sigma^{1 / 3} R_{\mathrm{L}}
$$

Therefore, our theory predicts effective particle acceleration in the region of the light cylinder up to energies corresponding to the Lorentz factor $10^{4}-10^{6}$. Clearly, such an acceleration is only possible within the fast magnetosonic surface, $r<r_{\mathrm{f}}$; as was noted, if the magnetized wind is free to reach the fast magnetosonic surface, then the longitudinal current is comparable in value to the Goldreich current.

Clearly, the effective acceleration of particles should result in the generation of hard radiation, which, in principle, could be detected. In [3], the synchrotron losses of accelerated particles are estimated, and both the total energy release and the energy range of radiation are shown to depend very strongly on the period $P$ of the pulsar. The energies of emitted photons can reach several tens of $\mathrm{MeV}$ only in the case of the youngest pulsars (Crab, Vela), while the radiation of most radio pulsars due to the synchrotron mechanism must lie in the optical range. The energy released in all ranges has turned out to be quite low, which has allowed observing these sources with the aid of existing detectors.

On the other hand, as is well known, another important channel in which energy is released and which is capable of resulting in the production of $\gamma$-quanta of even higher energies is the inverse Compton scattering of thermal X-ray photons emitted from the surface of a neutron star. This process has recently been regarded as the main process for the generation of photons of energies in the $\mathrm{TeV}$ range for the widest class of objects, such as active galactic nuclei $[79,80]$, galactic sources in the $\mathrm{TeV}$ range [81], and, naturally, radio pulsars [82]. In those cases where the 'central engine' is indeed a rapidly rotating neutron star, the Lorentz factor of electrons (or positrons) necessary for shifting the observed photons and soft $\gamma$-quanta toward the $\mathrm{TeV}$ energies corresponds precisely to values $\gamma=10^{4}-10^{5}$. Thus, for the pulsar B1259-63 (which is in a double system containing a Bestar), observations agree best with the value $\gamma \sim 10^{4}$ [83]. As can be readily estimated from relations (21) and (22), precisely this value is the characteristic value of the magnetization parameter $\sigma$ for B1259-63 .

We especially draw attention to the work of the group of F Aharonian published in Nature [82], the title of which is precisely the following: "Abrupt acceleration of a 'cold' ultrarelativistic wind from the Crab pulsar." It is shown in Ref. [82], for example, that the observed intensity of $\mathrm{TeV}$ photons can be explained, if a rapid acceleration of particles occurs at distances of the order of $30 R_{\mathrm{L}}$ as a result of which the particles acquire energies corresponding to Lorentz factors $\gamma \approx 10^{6}$. As was 
noted above, the value $\gamma \sim 10^{6}$ corresponds precisely to estimate (22) for the magnetization parameter $\sigma$ of the Crab pulsar. Moreover, the scale of $30 R_{\mathrm{L}}$ is certainly smaller than the radius of the fast magnetosonic surface $r_{\mathrm{f}} \approx 100 R_{\mathrm{L}}$ (see Eqn 23).

A detailed comparison of theoretical predictions and observational data is beyond the scope of this article, however 5 . Nevertheless, it must be noted that after it became clear that the existence of a large potential difference $\Delta V$ between the magnetic field lines in the magnetized wind does not lead directly to any effective particle acceleration up to ultra-high energies $\mathcal{E}_{\max } \sim \lambda \mathcal{E}_{\mathrm{e}}$ (see, e.g., Ref. [84]), the possibility of direct electrostatic acceleration of particles is not being taken into consideration (see, e.g., Ref. [85]). However, as was shown above, this process could well be realized in the case where, for some reason or other, the longitudinal current flowing in the magnetosphere of a compact object is quite small.

\section{Theory of radio emission}

\subsection{Formulation of the problem}

As is well known, one of the methods for determining the instability increment of waves in a plasma consists in analyzing the dispersion equation, for which it is necessary to determine the dielectric permittivity tensor of the medium. The procedure for calculating the dielectric permittivity tensor of an inhomogeneous anisotropic plasma in the approximation of geometric optics on the basis of the standard approach, expounded, for example, in Ref. [86], is described in detail in [2]. In the same work, a study is presented of the collective interaction in which electromagnetic waves associated with curvature radiation are simultaneously amplified by the Cherenkov mechanism. We emphasize that this effect is absent in the vacuum. Most likely because the calculation procedure is quite complicated, erroneous assertions have been made in a number of publications [87-89]. The objections put forward in Ref. [89] were later withdrawn [90] by the author. As regards Refs [87, 88], which are still cited in papers on the relevant topic, they contain numerous arithmetic errors, which have all been revealed and described in detail in Ref. [42].

In addition, another method for dealing with the problem of collective curvature radiation was proposed in [36$38,91]$. In these studies, a model problem, which could be solved 'exactly', was considered in a cylindrical geometry. The magnetic field in this problem is assumed to be precisely circular, while the relativistic plasma moves along the circular magnetic field lines owing to the centrifugal drift, $u=c \rho_{c} / \rho_{0} \ll c$, directed parallel to the cylindrical axis. Here, $\rho_{c}=c / \omega_{\mathrm{c}}$ is the cyclotron radius and $\rho_{0}$ is the curvature radius. But as we now show, this approach cannot be used in analyzing the collective curvature radiation either (in more detail see [96]).

\footnotetext{
${ }^{5}$ In our opinion, the distance from the acceleration region to the light cylinder amounting to $30 R_{\mathrm{L}}$ for the Crab pulsar may be overestimated.
}

As in [36-38], we consider the electromagnetic fields in the wave to be of the form

$$
[\mathbf{E}, \mathbf{B}]=[\mathbf{E}(\rho), \mathbf{B}(\rho)] \times \exp \left\{-i \omega t+i s \varphi+i k_{z} z\right\},
$$

where, $\omega$ is the wave frequency, $s$ is an integer defining the azimuthal component $k_{\varphi}=s / \rho$ of the wave vector, and $k_{z}$ is the component of the wave vector parallel to the cylinder axis. In the approach considered, the amplitudes are assumed to depend only on the coordinate $\rho$. Moreover, not the vectors $\mathbf{E}$ and $\mathbf{B}$, but their cylindrical components $(E, B)_{\rho},(E, B)_{\varphi}$ and $(E, B)_{z}$ depend only on the cylindrical radius $\rho$. This means that the polarization in the wave follows the magnetic field, turning from one point to another, which is possible only in the case of a well-defined boundary condition, for instance, for a system inside a metal cylinder. Under these conditions, we arrive at a one-dimensional problem, which can indeed be readily solved. However, as is not difficult to show, the wave considered within such an approach has nothing to do with curvature radiation.

To show this, we consider a particle moving along a circular trajectory of radius $\rho_{0}$ with a constant velocity $v$. Such motion corresponds to an infinite magnetic field. Then the emitted energy is equal to the work performed by the field of the wave on the electric current of the particle. The electric current is given by

$$
\mathbf{j}=e v \delta(\varphi-\Omega t) \delta(z) \frac{\delta\left(\rho-\rho_{0}\right)}{\rho} \mathbf{e}_{\varphi}
$$

where $\Omega=v / \rho_{0}$. For the polarization chosen,

$$
\int \mathbf{j} \mathbf{E} \mathrm{d} \mathbf{r}=e v E_{\varphi}\left(\rho_{0}\right) \exp \{-i \omega t+i s \Omega t\}
$$

It hence follows that radiation is possible only when $\omega=s \Omega=k_{\varphi} v$. But this is the condition for Cherenkov, but not curvature, radiation. A wave with such polarization cannot be generated by the curvature mechanism. The difference between curvature and Cherenkov waves is that the interaction time of bremsstrahlung and the irradiating particle is finite. A freely propagating wave with a nearly constant polarization deviates from the direction of motion of the particle. As a result, a nonzero projection of the electric field of the wave arises in the direction of the electric current of the particle, i.e., an exchange of energy between the wave and the particle becomes possible. The process lasts a finite time $\tau$, which can be found from the condition $\tau(\omega-\mathbf{k v}) \simeq 1$. For relativistic particles $(v \simeq c)$ we have $\tau=\left(\rho_{0}^{2} / \omega c^{2}\right)^{1 / 3} \simeq \rho_{0} / c \gamma$.

\subsection{Polarization of the curvature wave}

It is well known that the radiation field of the electric current $\mathbf{j}$ and of the charge density $\rho_{\mathrm{e}}$ of a moving particle 
of charge $e$ is described by retarded potentials [43]

$$
\begin{aligned}
& \mathbf{A}=\frac{1}{c} \int \frac{\mathbf{j}\left(t^{\prime}\right)}{R} \mathrm{~d} \mathbf{r}, \\
& \Phi=\int \frac{\rho_{\mathrm{e}}\left(t^{\prime}\right)}{R} \mathrm{~d} \mathbf{r},
\end{aligned}
$$

where $t^{\prime}=t-R / c$ is the so-called retarded time and $R$ is the distance from the charge to the observer who is at the point with coordinates $(\rho, \varphi, z)$ at the moment $t^{\prime}$,

$$
\begin{aligned}
R & =\left[\rho^{2}+z^{2}+\rho_{0}^{2}-2 \rho \rho_{0} \cos \left(\varphi^{\prime}-\varphi\right)\right]^{1 / 2}, \\
\varphi^{\prime} & =\Omega t^{\prime} .
\end{aligned}
$$

We now introduce the Fourier transform of potentials (27) and (28) with respect to time:

$$
\begin{aligned}
& \mathbf{A}_{\omega}=\frac{1}{2 \pi} \int \mathbf{A}(t) \exp \{i \omega t\} \mathrm{d} t, \\
& \Phi_{\omega}=\frac{1}{2 \pi} \int \Phi(t) \exp \{i \omega t\} \mathrm{d} t .
\end{aligned}
$$

It is convenient in what follows to pass from integration over $t$ to integration over the retarded time $t^{\prime}$, and then over the angle $\left(\varphi^{\prime}-\varphi\right)$. As a result, in Cartesian coordiates $(x, y, z)$ we obtain the vector potential $\mathbf{A}$ and the scalar potential $\Phi$ in the form

$$
\left[\mathbf{A}_{\omega} ; \Phi_{\omega}\right]=\frac{e \rho_{0}}{2 \pi c} \exp \{i \omega \varphi / \Omega\}\left[-K_{s} ; K_{c} ; 0 ; \frac{c}{v} K_{0}\right],
$$

where $K_{0}, K_{\mathrm{s}}$, and $K_{\mathrm{c}}$ are functions of only the coordinates $\rho$ and $z$ :

$$
\begin{aligned}
K_{0} & =\int \frac{\exp \left\{i \omega\left(R / c+\Omega^{-1}\right) \alpha\right\}}{R+v \rho \sin \alpha / c} \mathrm{~d} \alpha, \\
K_{\mathrm{s}} & =\int \frac{\exp \left\{i \omega\left(R / c+\Omega^{-1}\right) \alpha\right\} \sin \alpha}{R+v \rho \sin \alpha / c} \mathrm{~d} \alpha, \\
K_{\mathrm{c}} & =\int \frac{\exp \left\{i \omega\left(R / c+\Omega^{-1}\right) \alpha\right\} \cos \alpha}{R+v \rho \sin \alpha / c} \mathrm{~d} \alpha, \\
R & =\left(\rho^{2}+z^{2}+\rho_{0}^{2}-2 \rho \rho_{0} \cos \alpha\right)^{1 / 2}
\end{aligned}
$$

We stress that expression (33) is valid not only in the wave zone but also at any point $\mathbf{r}$. The dependence on the angle $\varphi$ is given by the factor $\exp \{i \omega \varphi / \Omega\}$. Hence, owing to the periodicity in the angle $\varphi$ we simply obtain $\omega=s \Omega$.

An important fact follows from relation (33): the field of the irradiated wave is a superposition of three harmonics: $s, s-1$ and $s+1$. For example, we present the following expression for the azimuthal component of the electric field $E_{\varphi \omega}$ :

$$
\begin{gathered}
E_{\varphi \omega}=\frac{i \omega}{v}\left(-\frac{\rho_{0}}{\rho} \Phi_{\omega}+\frac{v}{c} A_{\varphi \omega}\right)= \\
-i \frac{e \rho_{0} \omega}{2 \pi v^{2}} \exp \{i s \varphi\}\left[\frac{\rho_{0}}{\rho} K_{0}-\frac{v^{2}}{c^{2}}\left(K_{\mathrm{s}} \sin \varphi+K_{\mathrm{c}} \cos \varphi\right)\right] .
\end{gathered}
$$

The first term in the right-hand part of (35), which is proportional to the scalar potential $\Phi$, is not significant in the wave zone $\rho \gg \rho_{0}$, but is significant in the vicinity of the particle trajectory, $\rho=\rho_{0}$. Owing to the presence of this term, a particle which is in resonance with one of the three harmonics, for instance, with $s$ th $(\omega=s \Omega)$, is knocked out of resonance by the adjacent harmonics $s \pm 1$. Here, the component $E_{\varphi \omega}$ changes sign in a time $\tau$. The synchronicity condition $1-\cos \Omega \tau \simeq 1-v^{2} / c^{2}=\gamma^{-2}$ determines the time as $\tau$

$$
\tau \simeq 1 / \Omega \gamma=\rho_{0} / c \gamma
$$

which coincides with the formation time of curvature radiation.

Hence, the emitted curvature wave comprises three harmonics, $s$ and $s \pm 1$, with a fixed relation between the amplitudes. Precisely this circumstance provides the curvature mechanism of radiation. The neighboring harmonics, $s \pm 1$, arise owing to modulation of the field of the emitted wave by the electric current of the particle whith the harmonic $s=1$. It can now be understood why simply dealing with collective curvature radiation in a cylindrical geometry with a single cylindrical harmonic does not reveal any significant amplification of waves $[36-38,91]$. The chosen polarization excludes the curvature radiation.

\subsection{Propagation of the triplet of har- monics}

It was shown in Section 3.2 that the curvature radiation of a single charged particle in the vacuum cannot be described by a single cylindrical harmonic $\exp \{i s \varphi\}$. In the problem of the collective curvature radiation of waves, modulation of the electric current of particles occurs at the same time as their excitation; therefore, the resonance azimuthal harmonic $s=\omega \rho / v_{\varphi}$ mixes with the harmonics of the modulation of the particle electric current, giving rise to harmonics with all possible values of $s$. Below, we show that all azimuthal harmonics contribute to the response of the medium to the electromagnetic field. But here, we show that propagation of the triplet $(s, s \pm 1)$ of cylindrical harmonics differs significantly from the propagation of a single harmonic, which is usually discussed in the literature.

We consider the simple cylindrical one-dimensional problem of the emission from a flux of cold relativistic plasma particles with charge $e$ and mass $m_{\mathrm{e}}$, which move in the $x y$ plane along an infinite azimuthal magnetic field $B_{0}=B_{\varphi}$. In this case, the particles can only move in the $\varphi$-direction with a velocity $v_{\varphi}$ at an arbitrary cylindrical radius $\rho$. We assume the nonperturbed particle number density $n_{\mathrm{e}}^{(0)}$ and velocity $v_{\varphi}^{(0)}$ to be constant, i.e., independent of the radius $\rho$. The electric current $\mathbf{j}$ then has only a component along $\varphi$, while the magnetic field of the wave has only the component $\left(B_{\rho}=B_{\varphi}=0\right)$. The electric field in the wave has two nonzero components, $E_{\rho}$ and $E_{\varphi}\left(E_{z}=0\right)$. 
The dependence of the wave field on the coordinates is given by

$\left[E_{\rho} ; E_{\varphi} ; B_{z}\right]=\left[E_{\rho}(\rho) ; E_{\varphi}(\rho) ; B_{z}(\rho)\right] \exp \{-i \omega t+i s \varphi\}$.

From the Maxwell equations, we obtain

$$
\begin{aligned}
& \frac{\mathrm{d} E_{\varphi}^{(\sigma)}}{\mathrm{d} \rho}=\frac{i \sigma}{\rho} E_{\rho}^{(\sigma)}-i \frac{\rho}{\sigma} \frac{\omega^{2}}{c^{2}} E_{\rho}^{(\sigma)}-\frac{E_{\varphi}^{(\sigma)}}{\rho}, \\
& \frac{\mathrm{d} E_{\rho}^{(\sigma)}}{\mathrm{d} \rho}=-i \frac{\sigma}{\rho} E_{\varphi}^{(\sigma)}+\frac{4 \pi}{\omega} \frac{\sigma}{\rho} j_{\varphi}^{(\sigma)}-\frac{E_{\rho}^{(\sigma)}}{\rho},
\end{aligned}
$$

where the index $\sigma$ corresponds to one of the three harmonics, $s$ or $s \pm 1$. For simplicity, we introduce the dimensionless variable $r=\rho \omega / c$, and the quantity

$$
\Lambda=\frac{\omega_{\mathrm{p}}^{2}}{\omega^{2} \gamma^{3}}
$$

where $\omega_{\mathrm{p}}=\left(4 \pi n_{\mathrm{e}} e^{2} / m_{e}\right)^{1 / 2}$ is the plasma frequency, $\gamma=\left(1-v_{\varphi}^{2} / c^{2}\right)^{-1 / 2}$ is the Lorentz factor of plasma particles, and

$$
G_{\sigma}=\frac{4 \pi j_{\varphi}^{(\sigma)}}{\Lambda \omega}
$$

is the dimensionless current. In the new variables, Eqns (38) and (39) become

$$
\begin{aligned}
\frac{\mathrm{d} E_{\varphi}^{(\sigma)}}{\mathrm{d} r} & =\frac{i \sigma}{r} E_{\rho}^{(\sigma)}-i \frac{r}{\sigma} E_{\rho}^{(\sigma)}-\frac{E_{\varphi}^{(\sigma)}}{r}, \\
\frac{\mathrm{d} E_{\rho}^{(\sigma)}}{\mathrm{d} r} & =-i \frac{\sigma}{r} E_{\varphi}^{(\sigma)}+\Lambda \frac{\sigma}{r} G_{\sigma}-\frac{E_{\rho}^{(\sigma)}}{r} .
\end{aligned}
$$

As was noted, we here consider the interaction of three waves, $s$ and $s \pm 1$. It is very important that the propagation of these waves is not independent: coupling between the waves is realized by means of the electrostatic field $\left[E_{\rho}(\rho) ; E_{\varphi}(\rho)\right] \exp \{i \varphi\}$ with the lowest azimuthal harmonic $s=1$. The electrostatic field turns out to be the result of nonlinear coupling of the high-frequency harmonics $s$ and $s \pm 1$. The propagation equations of the mode $s=1$ in the same notation have the form

$$
\begin{aligned}
\frac{\mathrm{d} E_{\varphi}}{\mathrm{d} r} & =\frac{i}{r} E_{\rho}-\frac{E_{\varphi}}{r}, \\
\frac{\mathrm{d} E_{\rho}}{\mathrm{d} r} & =-i \frac{1}{r} E_{\varphi}+\Lambda Z-\frac{E_{\rho}}{r},
\end{aligned}
$$

where $Z=4 \pi n_{\mathrm{e}} e c /(\Lambda \omega)$. We stress that the second term in the right-hand part of Eqn (42) is absent in Eqn (44) because the field proportional to $\exp (i \phi)$ is static $(\omega=0)$.

To determine the response of the medium to the electromagnetic fields, we can use the continuity equation and the Euler equation:

$$
\begin{aligned}
& \frac{\partial n_{\mathrm{e}}}{\partial t}+\nabla\left(n_{\mathrm{e}} \mathbf{v}\right)=0 \\
& \left(\frac{\partial}{\partial t}+\mathbf{v} \nabla\right) \mathbf{p}=e\left(\mathbf{E}+\left[\frac{\mathbf{v}}{c}, \mathbf{B}\right]\right) .
\end{aligned}
$$

It is easy to verify that only the $\varphi$-component of the Euler equation is significant, while the radial component provides the equilibrium configuration across the infinite magnetic field.

We represent the velocity of plasma particles and the flux number density in terms of the expansion in powers of the wave field amplitudes:

$$
\begin{aligned}
& v_{\varphi}=v_{\varphi}^{(0)}+\delta v_{\varphi}^{(1)}+\delta v_{\varphi}^{(2)}+\ldots \\
& n_{\mathrm{e}}=n_{\mathrm{e}}^{(0)}+\delta n_{\mathrm{e}}^{(1)}+\delta n_{\mathrm{e}}^{(2)}+\ldots
\end{aligned}
$$

The linear response can be readily found as

$$
\begin{aligned}
& n_{\mathrm{e}}^{(1)}=n_{\mathrm{e}}^{(0)} \frac{k v_{\varphi}^{(1)}}{\omega-k v_{\varphi}^{(0)}}, \\
& v_{\varphi}^{(1)}=i \frac{e E_{\varphi}}{m_{\mathrm{e}} \gamma^{3}\left(\omega-k v_{\varphi}^{(0)}\right)},
\end{aligned}
$$

where $k=s / \rho$. To find the nonlinear response of the medium, it is necessary to take the nonlinear relation between $\delta v_{\varphi}$ and $\delta p_{\varphi}$ into account:

$$
\delta p_{\varphi}=m_{\mathrm{e}} \gamma^{3} \delta v_{\varphi}-\frac{3}{2} m_{\mathrm{e}} v_{\varphi}^{(0)} \gamma^{5} \frac{\left(\delta v_{\varphi}\right)^{2}}{c^{2}}
$$

Straightforward calculation gives

$$
\begin{aligned}
G_{s} & =\frac{1}{1-s v_{\varphi}^{(0)} / r}\left[i \frac{E_{\varphi}^{s}}{1-s v_{\varphi}^{(0)} / r}+\alpha \frac{r}{v_{\varphi}^{(0)}}\right. \\
& \times\left(A_{s, s-1} \frac{E_{\varphi}^{s-1} E_{\varphi}^{1}}{1-(s-1) v_{\varphi}^{(0)} / r}\right. \\
& \left.\left.-A_{s, s+1} \frac{E_{\varphi}^{s+1} E_{\varphi}^{1 *}}{1-(s+1) v_{\varphi}^{(0)} / r}\right)\right], \\
G_{s-1} & =\frac{1}{1-(s-1) v_{\varphi}^{(0)} / r}\left[i \frac{E_{\varphi}^{s-1}}{1-(s-1) v_{\varphi}^{(0)} / r}\right. \\
& \left.-\alpha \frac{r}{v_{\varphi}^{(0)}} A_{s, s-1} \frac{E_{\varphi}^{s} E_{\varphi}^{1 *}}{1-s v_{\varphi}^{(0)} / r}\right], \\
G_{s+1} & =\frac{1}{1-(s+1) v_{\varphi}^{(0)} / r}\left[i \frac{1-(s+1) v_{\varphi}^{(0)} / r}{1-r}\right. \\
& \left.+\alpha \frac{r}{v_{\varphi}^{(0)}} A_{s, s+1} \frac{E_{\varphi}^{s} E_{\varphi}^{1}}{1-s v_{\varphi}^{(0)} / r}\right],
\end{aligned}
$$



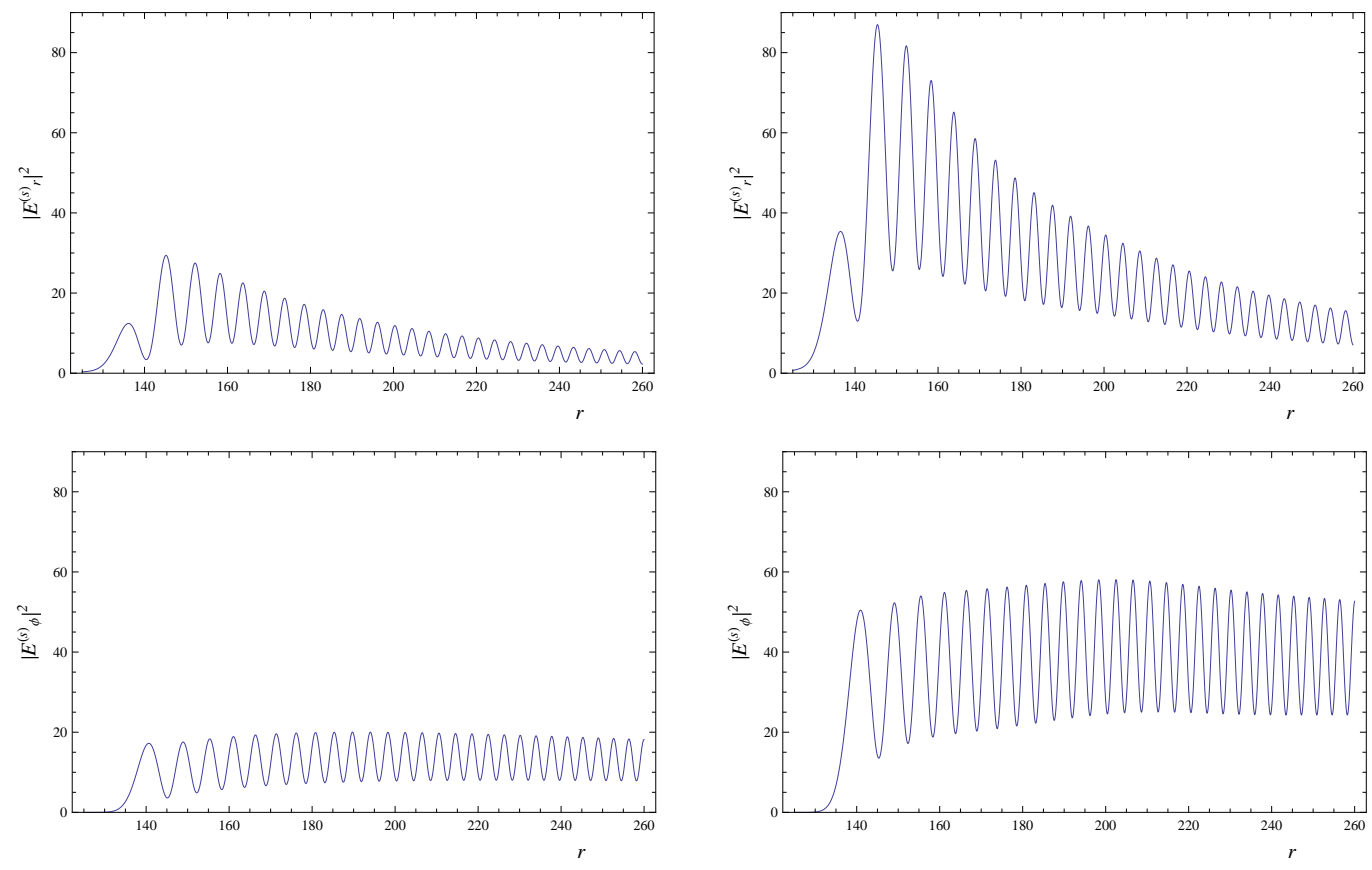

Figure 6: Wave amplification when the coupling the other harmonics is neglected (left), and when it is taken into account (right); $\Lambda=10^{-2}, \nu=1 \mathrm{GHz}, \gamma=5$, and $s=125$.

$$
\begin{aligned}
Z & =\frac{1}{\left(v_{\varphi}^{(0)}\right)^{2}}\left[i \frac{E_{\phi}^{1}}{1 / r}+\right. \\
& +\alpha\left(\frac{E_{\varphi}^{s+1} E_{\varphi}^{s *}}{\left(1-(s+1) v_{\varphi}^{(0)} / r\right)\left(1-s v_{\varphi}^{(0)} / r\right)}\right. \\
& \left.\left.+\frac{E_{\varphi}^{s} E_{\varphi}^{(s-1) *}}{\left(1-s v_{\varphi}^{(0)} / r\right)\left(1-(s-1) v_{\varphi}^{(0)} / r\right)}\right)\right] \\
A_{a, b} & =\frac{1}{1-a v_{\varphi}^{(0)} / r}+\frac{1}{1-b v_{\varphi}^{(0)} / r}-3 \gamma^{2}
\end{aligned}
$$

where $\alpha=e /\left(m_{e} c \gamma^{3} \omega\right)$ and the velocity of plasma particles is expressed in terms of the speed of light $c$. Similar quantities can be found in Ref. [3] for plane waves. The equations were analyzed numerically with the initial condition $E_{\varphi}^{(\sigma)}=-J_{\sigma}^{\prime}(r), E_{r}^{(\sigma)}=i \sigma J_{\sigma}(r) / r$, corresponding to the normal mode in the vacuum for a cylindrical geometry; here, $J_{\sigma}(r)$ is the Bessel function of order $\sigma$. The singularity in Eqns (53)-(56) was smoothed out, as usual, by adding a small term $+i \varepsilon$ to the resonance denominators in (50) and (51).

Equations (42)-(45) were numerically solved for $\sigma=s$ and $\sigma=s \pm 1$ at two different values of $G_{\sigma}$ and $Z$. In the first case, we neglected the nonlinear terms in (53)-(56), while the second case corresponded to the fully nonlinear problem. The results of calculations for both cases are presented in Fig. 6. To illustrate the influence of the nonlinear current better, we have chosen the amplitude of the modes $s-1$ and $s+1$ to be 20 times larger than the amplitude of the s mode. Actually, the $s$ mode couples to the entire continuum of modes, and hence the above model assumption is reasonable. Fig. 6 reveals $|E|^{2}$ to be 2.5 times larger in the fully nonlinear problem than when the nonlinear current is neglected.

Thus, we have shown that the triplet of cylindrical harmonics, better correponding to the curvature mechanism, is amplified more effectively than a single cylindrical harmonic. This means, inter alia, that the true polarization of collective curvature modes can only be obtained by calculating the dielectric permittivity tensor of the plasma flowing in a strong curved magnetic field. Here, the solution of the wave equations not only yields the dispersion relation for normal modes $\omega=\omega(\mathbf{k})$ but also determines their polarization. We note that initially, it is totally unclear what polarization corresponds to nonstable modes.

At first glance, the essentially nonlinear problem discussed above is not directly relevant to the amplification problem in the linear approximation. We included the nonlinearity only in order to examine the self-consistent coupling of modes $s$ and $s \pm 1$. Even in the case of a weak nonlinearity, the presence of adjacent modes $s \pm 1$ alters the amplification of the $s$ mode significantly. It is also absolutely clear that the coupling of harmonics $s \pm 1$ with the low-frequency harmonic $s=1$ results in the appearance of all possible azimuthal harmonics. 


\subsection{Calculation of the dielectric permit- ity operator: tivity tensor}

In this section, we show that the asymptotic form of the dielectric permittivity tensor obtained in Ref. [2] for large values of the magnetic field curvature radius $\rho_{0}$ can be found by straightforward summation of responses (50) and (51) to individual cylindrical modes. We first note that in the case of an infinite toroidal field, there is only a response to the toroidal component $E_{\varphi}$ of the wave electric field [92]. Here, we only consider the case of a stationary medium; therefore, the time dependence can be chosen to be of the form $\exp \{-i \omega t\}$.

Summation over all cylindrical modes yields

$$
D_{\varphi}(\rho, \varphi)=E_{\varphi}(\rho, \varphi)-\sum_{s=-\infty}^{\infty} E_{\varphi}(\rho, s) K(\rho, s) \exp \{i s \varphi\}
$$

where (see, e.g., Ref. [36])

$$
K(\rho, s)=\frac{4 \pi e^{2}}{\omega} \int \frac{v_{\varphi}}{\omega-s v_{\varphi} / \rho} \frac{\partial f^{(0)}}{\partial p_{\varphi}} \mathrm{d} p_{\varphi}
$$

and $f^{(0)}\left(p_{\varphi}\right)$ is the nonperturbed particle distribution function. Applying the Fourier transformation

$$
E_{\varphi}(\rho, s)=\frac{1}{2 \pi} \int_{0}^{2 \pi} E_{\varphi}\left(\rho, \varphi^{\prime}\right) \exp \left\{-i s \varphi^{\prime}\right\} \mathrm{d} \varphi^{\prime}
$$

and passing to a Cartesian coordinate system, we find

$$
\begin{array}{r}
D_{x}=E_{x}+\frac{1}{2 \pi} \int \frac{\rho^{\prime} \mathrm{d} \rho^{\prime} \mathrm{d} \varphi^{\prime}}{\rho^{\prime}} \sum_{s=-\infty}^{\infty} E_{\varphi}\left(\rho^{\prime}, \varphi^{\prime}\right) \delta\left(\rho-\rho^{\prime}\right) \\
\times K(\rho, s) \exp \left\{i s\left(\varphi-\varphi^{\prime}\right)\right\} \sin \varphi, \\
D_{y}=E_{y}-\frac{1}{2 \pi} \int \frac{\rho^{\prime} \mathrm{d} \rho^{\prime} \mathrm{d} \varphi^{\prime}}{\rho^{\prime}} \sum_{s=-\infty}^{\infty} E_{\varphi}\left(\rho^{\prime}, \varphi^{\prime}\right) \delta\left(\rho-\rho^{\prime}\right) \\
\times K(\rho, s) \exp \left\{i s\left(\varphi-\varphi^{\prime}\right)\right\} \cos \varphi .
\end{array}
$$

We choose a local coordinate sytem in the particle orbit plane with the $y$ axis directed along the magnetic field. From the expressions presented above, we obtain the components of the kernel of the dielectric permittiv-

$$
\begin{aligned}
\varepsilon_{y y}\left(\mathbf{r}, \mathbf{r}^{\prime}\right)=1 & -\frac{1}{2 \pi} \frac{1}{\rho^{\prime}} \sum_{s=-\infty}^{\infty} \delta\left(\rho-\rho^{\prime}\right) K(\rho, s) \\
& \times \exp \left\{i s\left(\varphi-\varphi^{\prime}\right)\right\} \cos \varphi \cos \varphi^{\prime} \\
\varepsilon_{y x}\left(\mathbf{r}, \mathbf{r}^{\prime}\right)= & \frac{1}{2 \pi} \frac{1}{\rho^{\prime}} \sum_{s=-\infty}^{\infty} \delta\left(\rho-\rho^{\prime}\right) K(\rho, s) \\
& \times \exp \left\{i s\left(\varphi-\varphi^{\prime}\right)\right\} \cos \varphi \sin \varphi^{\prime} \\
\varepsilon_{x y}\left(\mathbf{r}, \mathbf{r}^{\prime}\right)= & \frac{1}{2 \pi} \frac{1}{\rho^{\prime}} \sum_{s=-\infty}^{\infty} \delta\left(\rho-\rho^{\prime}\right) K(\rho, s) \\
& \times \exp \left\{i s\left(\varphi-\varphi^{\prime}\right)\right\} \sin \varphi \cos \varphi^{\prime} \\
\varepsilon_{x x}\left(\mathbf{r}, \mathbf{r}^{\prime}\right)=1 & -\frac{1}{2 \pi} \frac{1}{\rho^{\prime}} \sum_{s=-\infty}^{\infty} \delta\left(\rho-\rho^{\prime}\right) K(\rho, s) \\
& \times \exp \left\{i s\left(\varphi-\varphi^{\prime}\right)\right\} \sin \varphi \sin \varphi^{\prime}
\end{aligned}
$$

which determine the material relation

$$
D_{i}(\mathbf{r})=\int \varepsilon_{i j}\left(\mathbf{r}, \mathbf{r}^{\prime}\right) E_{j}\left(\mathbf{r}^{\prime}\right) \mathrm{d} \mathbf{r}^{\prime}
$$

We note that the kernel found satisfies the necessary symmetry property

$$
\varepsilon_{i j}\left(\mathbf{r}, \mathbf{r}^{\prime}, \omega\right)=\varepsilon_{j i}\left(\mathbf{r}^{\prime}, \mathbf{r},-\omega\right)
$$

resulting from the condition $K(r, s, \omega)=K(r,-s,-\omega)$.

Further, it is well known that for calculating the dielectric permittivity tensor, only the symmetrized form of $\varepsilon_{i j}(\omega, \mathbf{k}, \mathbf{r})$ must be used [93, 94]:

$$
\varepsilon_{i j}(\omega, \mathbf{k}, \boldsymbol{\eta} \rightarrow \mathbf{r})=\int \varepsilon_{i j}(\omega, \boldsymbol{\xi}, \boldsymbol{\eta}) \exp \{-i \mathbf{k} \boldsymbol{\xi}\} \mathrm{d} \xi
$$

Here, by definition, $\boldsymbol{\eta}=\left(\mathbf{r}+\mathbf{r}^{\prime}\right) / 2$ and $\boldsymbol{\xi}=\mathbf{r}-\mathbf{r}^{\prime}$. It is important to note that only this tensor correctly describes the interaction between waves and particles in a medium with slowly varying parameters (see, e.g., Refs $[35,86]$; in problems dealing with cosmological plasma, this approach was applied in Ref. [95]).

Substituting the components of the kernel, we now 
obtain

$$
\begin{aligned}
& \varepsilon_{x x}(\omega, \mathbf{k}, \boldsymbol{\eta})=1-\frac{1}{2 \pi} \int \mathrm{d} \boldsymbol{\xi} \exp \{-i \mathbf{k} \boldsymbol{\xi}\} \frac{1}{|\boldsymbol{\eta}-\boldsymbol{\xi} / 2|} \times \\
& \sum_{s=-\infty}^{\infty} \delta(|\boldsymbol{\eta}+\boldsymbol{\xi} / 2|-|\boldsymbol{\eta}-\boldsymbol{\xi} / 2|) K(|\boldsymbol{\eta}+\boldsymbol{\xi} / 2|, s) \times \\
& \exp \left\{i s\left(\varphi-\varphi^{\prime}\right)\right\} \sin \varphi \sin \varphi^{\prime}, \\
& \varepsilon_{x y}(\omega, \mathbf{k}, \boldsymbol{\eta})=\frac{1}{2 \pi} \int \mathrm{d} \boldsymbol{\xi} \exp \{-i \mathbf{k} \boldsymbol{\xi}\} \frac{1}{|\boldsymbol{\eta}-\boldsymbol{\xi} / 2|} \times \\
& \sum_{s=-\infty}^{\infty} \delta(|\boldsymbol{\eta}+\boldsymbol{\xi} / 2|-|\boldsymbol{\eta}-\boldsymbol{\xi} / 2|) K(|\boldsymbol{\eta}+\boldsymbol{\xi} / 2|, s) \times \\
& \exp \left\{i s\left(\varphi-\varphi^{\prime}\right)\right\} \sin \varphi \cos \varphi^{\prime}, \\
& \varepsilon_{y x}(\omega, \mathbf{k}, \boldsymbol{\eta})=\frac{1}{2 \pi} \int \mathrm{d} \boldsymbol{\xi} \exp \{-i \mathbf{k} \boldsymbol{\xi}\} \frac{1}{|\boldsymbol{\eta}-\boldsymbol{\xi} / 2|} \times \\
& \sum_{s=-\infty}^{\infty} \delta(|\boldsymbol{\eta}+\boldsymbol{\xi} / 2|-|\boldsymbol{\eta}-\boldsymbol{\xi} / 2|) K(|\boldsymbol{\eta}+\boldsymbol{\xi} / 2|, s) \times \\
& \exp \left\{i s\left(\varphi-\varphi^{\prime}\right)\right\} \cos \varphi \sin \varphi^{\prime}, \\
& \varepsilon_{y y}(\omega, \mathbf{k}, \boldsymbol{\eta})=1-\frac{1}{2 \pi} \int \mathrm{d} \boldsymbol{\xi} \exp \{-i \mathbf{k} \boldsymbol{\xi}\} \frac{1}{|\boldsymbol{\eta}-\boldsymbol{\xi} / 2|} \times \\
& \sum_{s=-\infty}^{\infty} \delta(|\boldsymbol{\eta}+\boldsymbol{\xi} / 2|-|\boldsymbol{\eta}-\boldsymbol{\xi} / 2|) K(|\boldsymbol{\eta}+\boldsymbol{\xi} / 2|, s) \times \\
& \exp \left\{i s\left(\varphi-\varphi^{\prime}\right)\right\} \cos \varphi \cos \varphi^{\prime}
\end{aligned}
$$

The angles $\varphi$ and $\varphi^{\prime}$ in expressions (69)-(72) are functions of the polar angles $\alpha_{\eta}$ and $\alpha_{\xi}$ of the vectors $\boldsymbol{\eta}$ and $\boldsymbol{\xi}$

$$
\begin{aligned}
\sin \varphi & =\frac{|\boldsymbol{\eta}| \sin \alpha_{\eta}+(|\boldsymbol{\xi}| / 2) \sin \alpha_{\xi}}{|\boldsymbol{\eta}+\boldsymbol{\xi} / 2|} \\
\cos \varphi^{\prime} & =\frac{|\boldsymbol{\eta}| \cos \alpha_{\eta}-(|\boldsymbol{\xi}| / 2) \cos \alpha_{\xi}}{|\boldsymbol{\eta}-\boldsymbol{\xi} / 2|}
\end{aligned}
$$

As a result, the integration in (69)-(72) reduces to integration over the components of the vector $\boldsymbol{\xi}$ that are perpendicular to $\boldsymbol{\eta}$. On the other hand, the the delta function in relations (69)-(72) are given by

$$
\begin{aligned}
& \delta(\ldots)=\frac{\delta(\theta-\pi / 2)}{(|\boldsymbol{\eta}+\boldsymbol{\xi} / 2|-|\boldsymbol{\eta}-\boldsymbol{\xi} / 2|)_{\theta}^{\prime}} \\
& +\frac{\delta(\theta+\pi / 2)}{(|\boldsymbol{\eta}+\boldsymbol{\xi} / 2|-|\boldsymbol{\eta}-\boldsymbol{\xi} / 2|)_{\theta}^{\prime}},
\end{aligned}
$$

where $\theta$ is the angle between vectors $\boldsymbol{\eta}$ and $\boldsymbol{\xi}$. Therefore, the integration over the angles is carried out in a trivial manner. Finally, performing the transition $\boldsymbol{\eta} \rightarrow \mathbf{r}$ we obtain $\cos \alpha_{\eta} \rightarrow \cos \alpha_{r}=1$. In accordance with (75), we can therefore write $(\mathbf{k} \boldsymbol{\xi})=k_{\|}|\boldsymbol{\xi}|$, where $k_{\|}$is the component of the wave vector directed along the magnetic field.

The property of the final result being independent of $k_{\perp}$ is very important. Precisely it provides the same symmetry property as in a homogeneous medium [42]:

$$
\varepsilon_{i j}(-\omega,-\mathbf{k},-\mathbf{B}, \mathbf{r})=\varepsilon_{j i}(\omega, \mathbf{k}, \mathbf{B}, \mathbf{r}) .
$$

If transformation (68) is neglected, the necessary symmetry cannot be obtained [36] (the authors of Ref. [36] explained the dependence of the dielectric tensor on $k_{\perp}$ by $k_{\perp}$ not being a Killing vector).

Finally, we use the Taylor expansion in $|\boldsymbol{\xi}|$ and the reduction of summation to a delta function:

$$
\begin{aligned}
& \sum(\ldots) \frac{1}{\omega|\boldsymbol{\eta}+\boldsymbol{\xi} / 2| / v_{\varphi}-s} \\
& \rightarrow i \pi \int(\ldots) \delta\left[s-\frac{\omega\left(|\boldsymbol{\eta}|^{2}+|\boldsymbol{\xi}|^{2} / 4\right)^{1 / 2}}{v_{\varphi}}\right] \mathrm{d} s .
\end{aligned}
$$

We then obtain

$$
\begin{gathered}
\varepsilon_{x x}=1-i \frac{8 \pi^{2} e^{2}}{\omega} \int F^{\prime \prime}(\kappa) \operatorname{fracv}_{\varphi} \omega \frac{\partial f^{(0)}}{\partial p_{\varphi}} \mathrm{d} p_{\varphi}, \\
\varepsilon_{x y}=-\varepsilon_{y x}=\frac{8 \pi^{2} e^{2}}{\omega} \int F^{\prime}(\kappa) \frac{\rho_{0}^{1 / 3} v_{\varphi}^{2 / 3}}{\omega^{2 / 3}} \frac{\partial f^{(0)}}{\partial p_{\varphi}} \mathrm{d} p_{\varphi}, \\
\varepsilon_{y y}=1-i \frac{8 \pi^{2} e^{2}}{\omega} \int F(\kappa) \frac{\rho_{0}^{2 / 3} v_{\varphi}^{1 / 3}}{\omega^{1 / 3}} \frac{\partial f^{(0)}}{\partial p_{\varphi}} \mathrm{d} p_{\varphi},
\end{gathered}
$$

where

$$
\begin{aligned}
F(\kappa) & =\frac{1}{\pi} \int_{0}^{+\infty} \exp \left\{i \kappa t+i t^{3} / 3\right\} \mathrm{d} t \\
\kappa & =\frac{2\left(\omega-k_{\|} v_{\varphi}\right)}{\omega^{1 / 3} v_{\varphi}^{2 / 3}} \rho_{0}^{2 / 3}
\end{aligned}
$$

the derivative of $F$ with respect to its argument $\kappa$ is indicated by a prime, and $\rho_{0}$ is the curvature radius of the magnetic field.

It can be readily verified that the condition $\kappa \gg 1$ is satisfied in the magnetosphere of radio pulsars owing to the large curvature radius $\rho_{0}$ of the magnetic field, and therefore we can use the asymptotic form of the function $F(\kappa)$

$$
F(\kappa) \approx \frac{i}{\pi \kappa}+\frac{2 i}{\pi \kappa^{4}}+\ldots
$$

Integrating by parts, we obtain the final result

$$
\varepsilon_{i j}=\left(\begin{array}{cc}
1-\frac{3}{2}\left\langle\frac{\omega_{\mathrm{p}}^{2} v_{\|}^{2}}{\gamma^{3} \rho_{0}^{2} \tilde{\omega}^{4}}\right\rangle & -i\left\langle\frac{\omega_{\mathrm{p}}^{2} v_{\|}}{\gamma^{3} \rho_{0} \tilde{\omega}^{3}}\right\rangle \\
i\left\langle\frac{\omega_{\mathrm{p}}^{2} v_{\|}}{\gamma^{3} \rho_{0} \tilde{\omega}^{3}}\right\rangle & 1-\left\langle\frac{\omega_{\mathrm{p}}^{2}}{\gamma^{3} \tilde{\omega}^{2}}\right\rangle
\end{array}\right),
$$

where, by definition, $\tilde{\omega}=\omega-\mathbf{k v}$, and the angular brackets stand both for averaging over the particle distribution function $f_{e^{+}, e^{-}}\left(p_{\varphi}\right)$ and for summation over the particle species:

$$
<(\ldots)>=\sum_{e^{+} e^{-}} \int(\ldots) f_{e^{+}, e^{-}}^{(0)}\left(p_{\varphi}\right) \mathrm{d} p_{\varphi} .
$$


We see that dielectric tensor (84) coincides exactly with the tensor obtained in Ref. [2] and that it is precisely the dielectric permittivity tensor that predicts the existence of unstable plasma-curvature modes. As expected, in the limit $\rho_{0} \rightarrow \infty$ the tensor found coincides with the tensor for homogeneous plasma. The nonzero components $\varepsilon_{x y}, \varepsilon_{y x}$ and $\delta \varepsilon_{x x}=\varepsilon_{x x}-1$ of $\varepsilon_{i j}$ in (84) for a finite curvature are due to the nonlocal response of the plasma to the electromagnetic field of the wave. The nonlocality parameter $\left(v_{\|} / \tilde{\omega}\right) / \rho_{0}$ is the ratio of the radiation formation length $l_{\mathrm{f}}=c \tau$ to the curvature radius. For the vacuum, $\tilde{\omega} \simeq \omega / \gamma^{2}$ and the length $v_{\|} / \tilde{\omega}$ coincides with the formation length of the curvature radiation $l_{\mathrm{f}}$.

It is important that the components $\varepsilon_{x y}=-\varepsilon_{y x}$ and $\delta \varepsilon_{x x}$ alter the wave polarization significantly. The relation between the components $E_{\varphi}$ and $E_{\rho}$ of the wave electric field, following from expression (84) for the dielectric permittivity tensor, is given by

$$
\left(\varepsilon_{x y}+n_{\rho} n_{\varphi}\right) E_{\varphi}+\left(\delta \varepsilon_{x x}+1-n_{\varphi}^{2}\right) E_{\rho}=0,
$$

where $n_{\rho}$ and $n_{\varphi}$ are dimensionless components of the wave vector $\mathbf{n}=\mathbf{k} c / \omega$. In the case of strictly azimuthal propagation (i.e., for $n_{\rho}=0$ ),

$$
E_{\varphi} \simeq \frac{\delta \varepsilon_{x x}}{\varepsilon_{x y}} E_{\rho} \simeq \frac{c}{\rho_{c} \tilde{\omega}} E_{\rho} .
$$

As a result, the electric field of the wave can perform negative work on the particle current $j_{\varphi}$, i.e., it can be excited. This property is violated if $\delta \varepsilon_{x x}=\varepsilon_{x y}=0$, whence it follows that $E_{\varphi}=0$.

Thus, as we have shown (also see Ref. [96]), a wave with a polarization $\left[E_{\rho}(\rho) ; E_{\varphi}(\rho)\right] \exp \{i s \varphi\}$, containing a single azimuthal harmonic $s$, satisfies only the Cherenkov radiation mechanism, while the emission of a charged particle in a circular magnetic field in the vacuum involves three azimuthal harmonics, $s$ and $s \pm 1$. This property provides an exit for the wave from the phase synchronism of the particle currrent. Further, in the case of collective curvature radiation, it was shown that the hydrodynamic model of plasma moving along an infinite magnetic field gives different results for wave amplification depending on the wave polarization. Consequently, there is no other way of finding the polarization of unstable modes except by calculating the response of the medium to the electromagnetic field, or, to be more precise, calculating the dielectric permittivity tensor. The correct procedure for calculation of the dielectric permittivity tensor via expansion into cylindrical modes is indicated above. The derived tensor coincides with the one obtained previously by another method [3].

In conclusion, we note that the unsuccessful attempts to find collective curvature radiation have led to the introduction of the term 'curvature-drift instability' [36]. As shown above, the polarization chosen in such an analysis, namely, a single azimuthal harmonic, only ensures the possibility of the Cherenkov amplification mechanism. In this case, the centrifugal drift of particles plays a decisive role. This is virtually a single curvature effect in such an analysis. The Cherenkov resonance, with account of the drift motion, at best provides a small wave amplification [37].

\subsection{Propagation theory of radio waves}

In this section, we make several comments concerning the most recent work on the theory of wave propagation in the magnetosphere of pulsars and on the formation problem of their average pulses. Over many years, a large number of polarimetric observations have been accumulated [97-100], and the hollow cone model, at least in its simplest realization, is not sufficient for their analysis. We recall that this model is based on the following three assumptions (see, e.g., Ref. [15]):

- the formation of polarization occurs at the point of emission;

- radio waves propagate along straight lines;

- cyclotron absorption can be neglected.

But all these assumptions turned out to be incorrect. It was shown in Ref. [101] that in the innermost regions of the magnetoshpere, the refraction of one of the normal modes is significant. After publication of the work of Mikhailovskii's group [102], it became clear that cyclotron absorption can significantly affect the radio emission intensity. The influence of the magnetosphere plasma on variation of the polarization of radio emission propagating in the internal regions of the magnetosphere also must not be neglected [103]. Here, the main point is the effect of limiting polarization, which consists in the following. The polarization of radio emission in the region of dense plasma satisfies the laws of geometric optics; therefore, the orientation of the polarization ellipse coincides with the magnetic field orientation in the picture plane. But the wave polarization in the vacuum region remains unchanged. Hence, there is a transition layer, after passing through which the polarization is no longer affected by the magnetospheric plasma. In the case of typical parameters of the pulsar magnetosphere, it turns out that the formation of polarization occurs not at the emission point but at a distance of about $\approx 0.1 R_{\mathrm{L}}$ from it $[104,105]$. Taking this effect into account should also explain the observed fraction of circular polarization of the order of $(5-10) \%$. Therefore, for a quantitative comparison of theoretical results on radio emission with observational data, it is necessary to have a consistent theory of radio wave propagation in the magnetoshpere.

At present, the theory of radio wave propagation in the magnetosphere of a pulsar can be considered to provide the necessary precision [106-110]. Four normal modes exist in the magnetosphere $[3,16]$. Two of them are plasma modes and two are electromagnetic, which are capable of departing from the magnetosphere. An extraordinary wave (the X-mode) with the polarization perpendicular to the magnetic field in the picture plane propagates along a straight line, while an ordinary wave (the Omode) undergoes refraction and deviates from the magnetic axis [101]. An important point here is that for 
typical magnetosphere parameters, refraction occurs at distances up to $\approx 0.01 R_{\mathrm{L}}$, i.e., it can be considered separately from the cyclotron absorption and the limiting polarization.

Based on the Kravtsov-Orlov method [109], we have developed [110] such a theory of wave propagation in a realistic pulsar magnetosphere taking corrections to the dipole magnetosphere into account (based on the results obtained by numerical simulation in Ref. [20]) together with the drift of plasma particles in crossed electric and magnetic fields and a realistic particle distribution function. The theory developed allows dealing with an arbitrary profile of the spatial plasma distribution, which may differ from the one in the hollow-cone model, because precisely the inhomogeneous plasma distribution leads to the characteristic 'patchy' directivity pattern [98].

The main result consists in the prediction of a correlation between the sign of the circular polarization (the Stokes parameter $V$ ) and the sign of the derivative of the change in the polarization of the position angle, p.a., along the profile, p.a., along the profile, $\mathrm{d} p . a . / \mathrm{d} \phi$, where $\phi$ is the phase of the radio pulse. For the ordinary mode, these signs must be opposite to each other, while for the extraordinary mode, they must coincide. As was noted, refraction of the ordinary wave leads to a deviation of beams from the rotation axis, and therefore the ordinary wave pattern should be broader than for the extraordinary wave. In the case of the ordinary mode, double radio emission profiles should mainly be observed, while single profiles should be observed in the case of the narrower extraordinary mode [3].

Observations also fully confirm this conclusion of the theory [111]. In reviews [99, 100], to perform an analysis, over 70 pulsars were chosen for which both the variation of the position angle and the sign of the circular polarization $V$ could be traced well (the results of the analysis are presented in the Table). In the case of opposite signs of the derivative $\mathrm{d} p . a . / \mathrm{d} \phi$ and the Stokes parameter $V$, the pulsar was placed in class $\mathrm{O}$, while in the case of identical signs, it was placed in class X. As can be seen from the Table, most of the pulsars exhibiting a doublepeaked (index D) profile indeed correspond to the ordinary wave, while most of the pulsars with single-peaked profiles (index $\mathrm{S}$ ) correspond to the extraordinary wave. Moreover, the average width of the radiation pattern at a $50 \%$ intensity level $W_{50}$ (normalized with account of different pulsar periods $P$ ) for $\mathrm{O}_{\mathrm{D}}$ pulsars is indeed about two times larger than the average width of the radiation pattern for $\mathrm{X}_{\mathrm{S}}$ pulsars. The existence of a certain number of pulsars of classes $\mathrm{O}_{\mathrm{S}}$ and $\mathrm{X}_{\mathrm{D}}$ should not give rise to surpise, because for central passage through the directivity pattern, independently of whether it corresponds to the $\mathrm{O}$-mode or to the $\mathrm{X}$-mode, a double-peaked profile should be observed, while for lateral passage, a singlepeaked profile should be observed.
Table 1: Statistics of pulsars with known circular polarization $V$ and variation of position angle p.a. The pulsar periods $P$ are expressed in seconds, and the window width $W_{50}$ in degrees.

\begin{tabular}{|c|c|c|c|c|}
\hline Profile & $\mathrm{O}_{\mathrm{S}}$ & $\mathrm{O}_{\mathrm{D}}$ & $\mathrm{X}_{\mathrm{S}}$ & $\mathrm{X}_{\mathrm{D}}$ \\
\hline Number & 6 & 23 & 45 & 6 \\
\hline$\sqrt{P} W_{50}$ & $6.8 \pm 3.1$ & $10.7 \pm 4.5$ & $6.5 \pm 2.9$ & $5.3 \pm 3.0$ \\
\hline
\end{tabular}

Another important result consists in the determination of the applicability range for the standard relation

$$
\text { p.a. }=\arctan \left(\frac{\sin \chi \sin \phi}{\sin \chi \cos \zeta \cos \phi-\sin \zeta \cos \chi}\right)
$$

describing variation of the position angle in the mean profile under the assumption of the validity of the hollowcone model (the absence of any absorption and the presence of a dipole magnetic field in the radiation region, precisely where the polarization is determined). Here, once again, $\chi$ is the inclination angle of the magnetic dipole to the rotation axis, $\zeta$ is the angle between the rotation axis and the direction toward the observer, and $\phi$ is the phase of the pulse. Accurately taking propagation effects into account has shown $[108,111]$ that such a variation of the position angle can be realized only under conditions of low plasma density or high mean particle energy. Significant deviations from prediction (88) were obtained in the case of quite reasonable parameters (for example, the multiplicity $\lambda \sim 10^{4}$ and the average Lorentz factor $\gamma \sim 50$ ), satisfying particle production models. We recall that precisely equation (88) has been for many years in estimating the pulsar inclination angle, which is a very important parameter for determining the structure of the magnetosphere. In the nearest future, we plan to perform a detailed comparison of observational data and theoretical predictions.

\section{Conclusion}

In our opinion, quite a sufficient number of arguments have been presented in this article to assert with confidence that the theory of the magnetosphere of radio pulsars and of the mechanism of their coherent radiation, developed by us in the 1980s [3], contains no internal contradictions. Moreover, as shown above, observational data obtained recently confirm the validity of the main conclusions of the theory. Therefore, with the most recent results [110] concerning the effects of wave propagation in the magnetosphere of a neutron star (see Section $3.5)$, the theory developed by us, unlike many others, permits making quantitative predictions concerning the evolution of neutron stars and properties of the observed radio emission.

Once again, we stress that the present article only concerns the theoretical foundation of our model of radio pulsars. A more detailed exposition of quantitative pre- 
dictions of the theory and their comparison with observational data must be discussed separately. This will be discussed elsewhere.

The authors are grateful to A V Gurevich for the valuable comments and support and to A Spitkovsky and A D Tchekhovskoy for the useful discussions. This work was partly supported by the Russian Foundation for Basic Research (grant 11-02-01021).

\section{References}

1. Beskin V S, Gurevich A V, Istomin Ya N Zh. Eksp. Teor. Fiz. 85401 (1983) [Sov. Phys. JETP 58235 (1983)]

2. Beskin V S, Gurevich A V, Istomin Ya N Astrophys. Space Sci. 146205 (1988)

3. Beskin V S, Gurevich A V, Istomin Ya N Physics of the Pulsar Magnetosphere (Cambridge: Cambridge Univ. Press, 1993)

4. Hewish A et al. Nature, 217709 (1968)

5. Ginzburg V L, Zheleznyakov V V Annu. Rev. Astron. Astrophys. 13511 (1975)

6. Ginzburg V L, Zheleznyakov V V, Zaitsev V V Usp. Fiz. Nauk 98201 (1969) [Sov. Phys. Usp. 12378 (1969)]

7. Kadomtsev B B, Kudryavtsev V S Pis'ma zh. Eksp. Teor. Fiz. 1361 (1971) [JETP Lett. 1342 (1971)]

8. Lominadze D G, Mikhailovskii A B, Sagdeev R Z Zh. Eksp. Teor. Fiz. 771951 (1979) [Sov. Phys. JETP 50927 (1979)]

9. Lominadze J G, Machabeli G Z, Usov V V Astrophys. Space Sci. 9019 (1983)

10. Goldreich P, Julian W H Astrophys. J. 157869 (1969)

11. Coppi B, Pegoraro F Ann. Physics 11997 (1979)

12. Melrose D Astrophys. J. 225557 (1978)

13. Mestel L Stellar Magnetism (New York: Oxford Univ. Press, 1999)

14. Ruderman M A, Sutherland P G Astrophys. J. 19651 (1975)

15. Manchester R N, Taylor J H Pulsars (San Francisco: W. H. Freeman, 1977)

16. Lyne A G, Graham-Smith F Pulsar Astronomy (Cambridge: Cambridge Univ. Press, 1998)

17. Sturrock P A Astrophys. J. 164529 (1971)

18. Bogovalov S V Astron. Astrophys. 3491017 (1999)

19. Lyubarsky Y, Kirk J G Astrophys. J. 547437 (2001)

20. Spitkovsky A Astrophys. J. 648 L51 (2006)

21. Kalapotharakos C, Contopoulos I Astron. Astrophys. 496495 (2009)

22. Michel F C Astrophys. J. 180207 (1973)

23. Mestel L, Wang Y-M Mon. Not. R. Astron. Soc. 188 799 (1979)

24. Contopoulos I, Kazanas D, Fendt Ch Astrophys. J. 511 351 (1999)

25. Goodwin S P et al. Mon. Not. R. Astron. Soc. 349213 (2004)

26. Gruzinov A Phys. Rev. Lett. 94021101 (2005)

27. Komissarov S S Mon. Not. R. Astron. Soc. 36719 (2006)

28. McKinney J Mon. Not. R. Astron. Soc. 368 L30 (2006) 29. Timokhin A N Mon. Not. R. Astron. Soc. 681055 (2006)
30. Beskin V S Usp. Fiz. Nauk 1691169 (1999) [Phys. Usp. 421071 (1999)]

31. Usov V V, in On the Present and Future of Pulsar Astronomy, 26th Meeting of the IAU, Prague, Czech Republic (2006)

32. Lyubarsky Yu AIP Conf. Proc. 98329 (2008)

33. Radhakrishnan V, Cooke D J Astrophys. Lett. 3225 (1969)

34. Beskin V S, Gurevich A V, Istomin Ya N Astrophys. Space Sci. 102 301(1984)

35. Beskin V S, Gurevich A V, Istomin Ya N Zh. Eksp. Teor. Fiz. 921277 (1987) [Sov. Phys. JETP 65715 (1987)] 36. Lyutikov M, Machabeli G, Blandford R Astrophys. J. 512804 (1999)

37. Kaganovich A, Lyubarsky Yu Astrophys. J. 7211164 (2010)

38. Larroche O, Pellat R Phys. Rev. Lett. 591104 (1987)

39. MelroseDB/.Astrophys. Astron. 16137 (1995)

40. Beskin V S, Gurevich A V, Istomin Ya N, Arons J Phys. Today 41 (10) 71 (1994)

41. Beskin V S, Gurevich A V, Istomin Ya N Phys. Rev. Lett. 61649 (1988)

42. Istomin Ya N Plasma Phys. Control. Fusion 361081 (1994)

43. Landau L D, Lifshitz E M Teoriya polya (Moscow: Nauka, 1973) The Classical Theory of Fields (Oxford: Butterworth-Heinemann, 1980)]

44. Henriksen R N, Norton J A Astrophys. J. 201719 (1975)

45. Mestel L, Panagi P, Shibata S Mon. Not. R. Astron. Soc. 309388 (1999)

46. Arons J Astrophys. J. 2481099 (1981)

47. Bai X-N, Spitkovsky A Astrophys. J. 7151282 (2010)

48. Beskin V S Usp. Fiz. Nauk 1801241 (2010) [Phys. Usp. 531199 (2010)]

49. Kramer M et al. Science 312549 (2006)

50. Camilo F et al. Astrophys. J. 74663 (2012); arXiv:l 111.5870

51. Beskin V S, Nokhrina E E Astophys. Space Sci. $\mathbf{3 0 8}$ 569 (2007)

52. Gurevich A V, Istomin Ya N Mon. Not. R. Astron. Soc. 3771663 (2007)

53. Li J, Spitkovsky A, Tchekhovskoy A Astrophys. J. 746 $24(2012)$

54. Istomin Ya N, Shabanova T V Astron. Zh. 84139 (2007) [Astron. Rep. 51119 (2007)]

55. Lyne A G, Manchester R N, Taylor J H Mon. Not. R. Astron. Soc. 213613 (1985)

56. Tauris T M, Manchester R N Mon. Not. R. Astron. Soc. 298625 (1998)

57. Young M D T et al. Mon. Not. R. Astron. Soc. 402 1317 (2010)

58. Beskin V S, Nokhrina E E Pis'ma Astron. Zh. 30754 (2004) [Astron. Lett. 30685 (2004)]

59. Beskin V S, Eliseeva S A Pis'ma Astron. Zh. 31290 (2005) [Astron. Lett. 31263 (2005)]

60. Eliseeva S A, Popov S B, Beskin V S, astro-ph/0611320 61. Lipunov V M, Postnov K A, Prokhorov M E Astron. Astophys. 310489 (1996)

62. Story S A, Gonthier P L, Harding A Astrophys. J. 671 
713 (2007)

63. Popov S B, Prokhorov M E Usp. Fiz. Nauk 1771179 (2007) [Phys Usp 501123 (2007)]

64. Michel F C Astrophys. J. 180 L133 (1973)

65. Mestel L Astrophys. Space Sci. 24289 (1973)

66. Lyubarskii Y E Pis'ma Astron. Zh. 1634 (1990) [Sov. Astron. Lett. 1616 (1990)]

67. Beskin V S Osecimmetrichnye statsionarnye techeniya $v$ astrofizike (Axisymmetric Flows in Astrophysics) (Moscow: Fizmatlit, 2005) [Beskin V S MHD Flows in Compact Astrophysical Objects (Heidelberg: Springer, 2010)]

68. Kennel C F, Coroniti F V Astrophys. J. 283694 (1984)

69. Barkov M V, Komissarov S S Mon. Not. R. Astron. Soc. 38528 (2008)

70. Tchekhovskoy A, McKinney J C, Narayan R Mon. Not. R. Astron. Soc. 388551 (2008)

71. Narayan R, McKinney J C, Farmer A F Mon. Not. R. Astron. Soc. 375548 (2007)

72. Beskin V S, Malyshkin L M Mon. Not. R. Astron. Soc. 298847 (1998)

73. Istomin Ya N, Sobyanin D N Zh. Eksp. Teor. Fiz. 136 458 (2009) [JETP 109393 (2009)]

74. Medin Z, Lai D Mon. Not. R. Astron. Soc. 4061379 (2010)

75. Timokhin A N Arons J Mon. Not. R. Astron. Soc. 429 20 (2013)

76. Beskin V S, Rafikov R R Mon. Not. R. Astron. Soc. 313433 (2000)

77. Michel F C Astrophys. J. 158727 (1969)

78. Daugherty J K, Harding A Astrophys. J. 252337 (1982)

79. Takahashi T et al. Astrophys. J. 470 L89 (1996)

80. Abdo A A et al. Astrophys. J. 7071310 (2009)

81. Bosch-Ramon V, Khangulyan D Int. J. Mod. Phys. 018347(2009)

82. Aharonian F A, Bogovalov S V, Khangulyan D Nature 482507 (2012)

83. Khangulyan D et al. Astrophys. J. 752 L17 (2012); arXiv:1107.4833

84. Berezinskii V S et al. Astrofizika kosmicheskikh luchei (Ed. V L Ginzburg) (Moscow: Nauka, 1990) [] Astrophysics of Cosmic Rays (Ed. V L Ginzburg) (Amsterdam: NorthHolland, 1990)]

85. Bogovalov S V et al. Mon. Not. R. Astron. Soc. 387 63 (2008)

86. Bernstein I B, Friedland L, in Basic Plasma Physics Vol. 1 (Handbook of Plasma Physics, Vol. 1, Eds A Galeev, R N Sudan) (Amsterdam: North-Holland, 1984) p. 367; 87. Machabeli G Z Plasma Phys. Control. Fusion 331227 (1991)

88. Machabeli G Z Plasma Phys. Control. Fusion 37177 (1995)

89. Nambu M Plasma Phys. Control. Fusion 31143 (1989) 90. Nambu M Phys. Plasmas 34325 (1996)

91. Asseo E, Pellat R, Sol H Astrophys. J. 266201 (1983)

92. Asseo E, Pellat R, Rosado M Astrophys. J. 239661 (1980)

93. Kadomtsev B B Kollektivnye yavleniya $v$ plazme (Collective phenomena in plasma) 2nd ed. (Moscow: Nauka, 1988) [Kadomtsev , in Reviews of Plasma Physics Vol. 22 (Ed. V D Shafranov) (New York: Kluwer Acad./Plenum
Publ., 2001) p. 1]

94. Bornatici M, Kravtsov Yu A Plasma Phys. Control. Fusion v 42255 (2000)

95. Dodin I Y, Fisch N J Phys. Rev. D 82044044 (2010)

96. Istomin Ya N, Philippov A A, Beskin V S Mon. Not. R. Astron. Soc. 422232 (2012)

97. Rankin J M Astrophys. J. 274333 (1983)

98. Rankin J M Astrophys. J. 352247 (1990)

99. Weltevrede P, Johnston S Mon. Not. R. Astron. Soc. 3911210 (2008)

100. Hankins T H, Rankin J M Astron. J. 139168 (2010)

101. Barnard J J, Arons J Astrophys. J. 302138 (1986)

102. Mikhailovskii A B et al. Pis'ma Astron. Zh. 8685 (1982) [Sov. Astron. Lett. 8369 (1982)]

103. Petrova S A, Lyubarskii Yu E Astron. Astrophys. 355 1168 (2000)

104. Cheng A F, Ruderman M A Astrophys. J. 229348 (1979)

105. Barnard J J Astrophys. J. 303280 (1986)

106. Petrova S A Mon. Not. R. Astron. Soc. 3681764 (2006)

107. Wang C, Lai D, Han J Mon. Not. R. Astron. Soc. 403 569 (2010)

108. Beskin V S, Philippov A A, arXiv: 1101.5733

109. Kravtsov Yu A, Orlov Yu I Geometricheskaya optika neodnorodnykh sred (Moscow: Nauka, 1980) [Geometrical Optics of Inhomogeneous Media (Berlin: Springer-Verlag, 1990)]

110. Beskin V S, Philippov A A Mon. Not. R. Astron. Soc. 425814 (2012)

111. Andrianov A S, Beskin V S Pis'ma Astron. Zh. 36260 (2010) [Astron. Lett. 36248 (2010)] 